\title{
TLR2 senses the SARS-CoV-2 envelope protein to produce inflammatory cytokines
}

\author{
Min Zheng', Rajendra Karki ${ }^{1}$, Evan Peter Williams², Dong Yang ${ }^{3}$, Elizabeth Fitzpatrick ${ }^{3}$, \\ Peter Vogel ${ }^{4}{ }^{4}$, Colleen Beth Jonsson ${ }^{2}$ and Thirumala-Devi Kanneganti ${ }^{1}{ }^{凶}$
}

\begin{abstract}
The innate immune response is critical for recognizing and controlling infections through the release of cytokines and chemokines. However, severe pathology during some infections, including SARS-CoV-2, is driven by hyperactive cytokine release, or a cytokine storm. The innate sensors that activate production of proinflammatory cytokines and chemokines during COVID-19 remain poorly characterized. In the present study, we show that both TLR2 and MYD88 expression were associated with COVID-19 disease severity. Mechanistically, TLR2 and Myd88 were required for $\beta$-coronavirus-induced inflammatory responses, and TLR2-dependent signaling induced the production of proinflammatory cytokines during coronavirus infection independent of viral entry. TLR2 sensed the SARS-CoV-2 envelope protein as its ligand. In addition, blocking TLR2 signaling in vivo provided protection against the pathogenesis of SARS-CoV-2 infection. Overall, our study provides a critical understanding of the molecular mechanism of $\beta$-coronavirus sensing and inflammatory cytokine production, which opens new avenues for therapeutic strategies to counteract the ongoing COVID-19 pandemic.
\end{abstract}

$\beta$ Coronaviruses belong to the family Coronaviridae and are positive-stranded enveloped RNA viruses. Several members of the $\beta$-coronavirus genus have emerged to cause zoonotic epidemic infections this century, including severe acute respiratory syndrome coronavirus 1 (SARS-CoV-1) and Middle East respiratory syndrome (MERS) coronavirus (MERS-CoV) ${ }^{1}$. The currently circulating SARS-CoV-2, which is responsible for the ongoing pandemic of coronavirus disease 2019 (COVID-19), is also a member of the $\beta$-coronavirus genus ${ }^{2}$.

$\beta$-Coronavirus infection leads to robust proinflammatory cytokine release, including tumor necrosis factor (TNF)- $\alpha$, interleukin (IL)-6, IL- $1 \beta$ and interferon (IFN) $-\gamma^{3-6}$. Although these cytokines are a critical component of the innate immune response and aid in clearing viral infections, the dysregulated release of proinflammatory cytokines can lead to a cytokine storm, which causes severe damage to host tissues and organs by inducing inflammatory cell death $^{3,6,7}$. COVID-19 has caused more than 3.2 million deaths globally to date, and increased levels of proinflammatory cytokines are directly associated with the pathogenesis ${ }^{8}$. A lack of fundamental understanding of the underlying pathways driving this dysregulated cytokine release has hampered the development of targeted therapeutic strategies. A recent study discovered the molecular mechanisms linking cytokine storm to mortality, showing that TNF- $\alpha$ and IFN- $\gamma$ are the key factors among the cytokines that are increased in patients with COVID-19. These two specific cytokines act synergistically to activate robust inflammatory cell death (PANoptosis), thereby causing tissue and organ damage and lethality ${ }^{6}$. However, the host factors that control the expression of inflammatory cytokines, including TNF- $\alpha$ and IFN- $\gamma$, during coronavirus infection are largely unknown.

The host innate immune system can recognize pathogenassociated molecular patterns (PAMPs) via pattern recognition receptors (PRRs) during infection to induce inflammatory responses to eliminate pathogens ${ }^{9}$. The five PRR families identified to date include toll-like receptors (TLRs), nucleotide-binding oligomerization domain (NOD)-like receptors (NLRs), retinoic acid-inducible gene-I (RIG-I)-like receptors (RLRs), C-type lectin receptors (CLRs) and the absent in melanoma 2 (AIM2)-like receptors (ALRs). PAMPs can include moieties such as cell wall components of pathogens (lipopolysaccharide (LPS) and lipoproteins), glycans, conserved proteins such as flagellin or pathogenic nucleic acids, including RNA and DNA; these moieties are generally conserved among pathogenic species but are distinct from host components ${ }^{10}$. Several PRRs have been reported to be involved in sensing $\beta$-coronavirus infection, including melanoma differentiation-associated protein 5 (MDA5 (ref. ${ }^{11}$ )), TLR7 (refs. ${ }^{12,13}$ ) and NLR family pyrin domain containing 3 (NLRP3 (ref. ${ }^{5}$ )). MDA5 contributes to mouse hepatitis virus (MHV)-induced type I IFN expression, but plays an inhibitory role in inflammatory cytokine expression in vivo ${ }^{11}$. TLR7 is required for MERS-CoVtriggered type I IFN induction in vivo and for MHV-induced type I IFN activation in plasmacytoid dendritic cells ${ }^{12,13}$, whereas the role of TLR7 in $\beta$-coronavirus-induced inflammatory cytokine release has not been examined. NLRP3 activation leads to the release of the proinflammatory cytokines IL-1 $\beta$ and IL-18 in response to $\beta$-coronavirus infection ${ }^{5}$. Myd88, a TLR adapter protein, is required for the production of other inflammatory cytokines, such as TNF- $\alpha$ and IL-6, after $\beta$-coronavirus infection ${ }^{14}$. However, the sensors upstream of Myd88 that control the activation of the inflammatory signaling pathway remain unclear during coronavirus infection.

In the present study, we screened several innate sensors upstream of Myd88 to determine the receptors required for the production of proinflammatory cytokines after $\beta$-coronavirus infection and to understand how these critical cytokines are produced, particularly given the pathogenic role of TNF- $\alpha$ and IFN- $\gamma$

'Department of Immunology, St Jude Children's Research Hospital, Memphis, TN, USA. ²Department of Microbiology, Immunology, \& Biochemistry, University of Tennessee Health Science Center, Memphis, TN, USA. ${ }^{3}$ UTHSC Regional Biocontainment Laboratory, University of Tennessee Health Science Center, Memphis, TN, USA. ${ }^{4}$ Animal Resources Center and Veterinary Pathology Core, St Jude Children's Research Hospital, Memphis, TN, USA.

凶e-mail: Thirumala-Devi.Kanneganti@StJude.org 
a

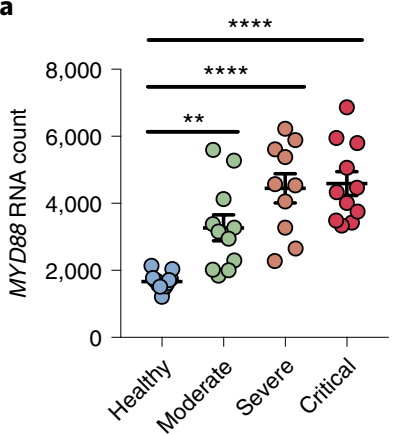

d

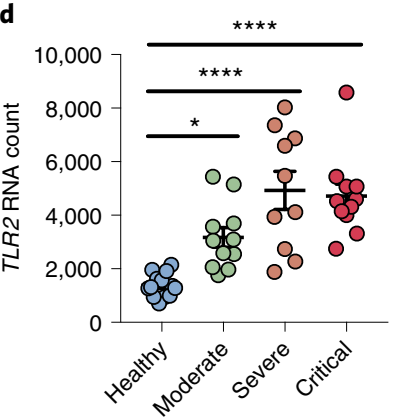

g

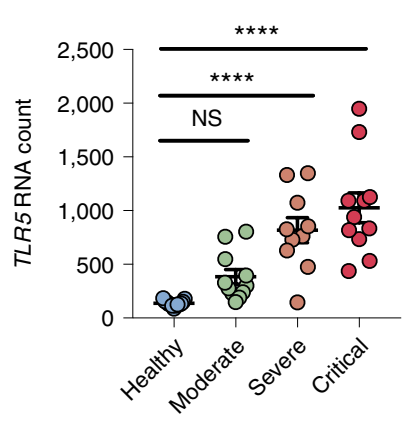

b

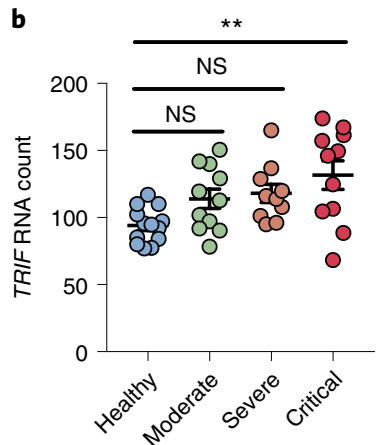

e

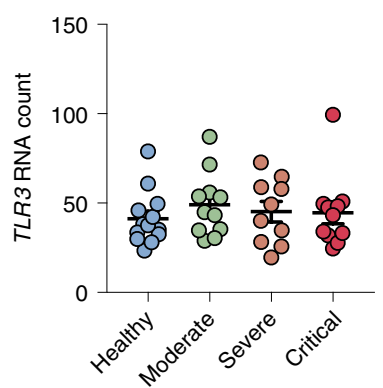

h

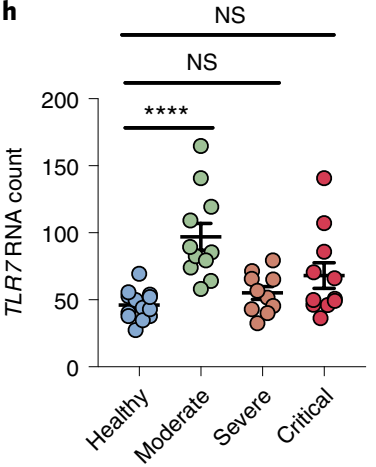

c

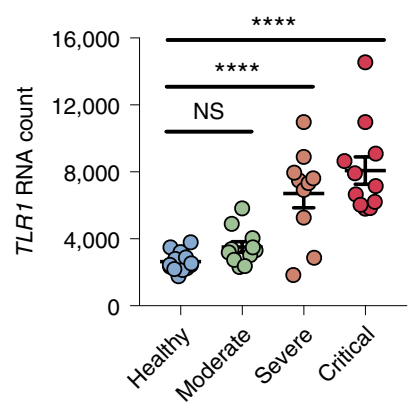

f

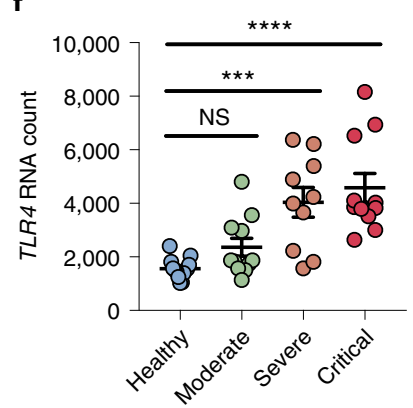

i

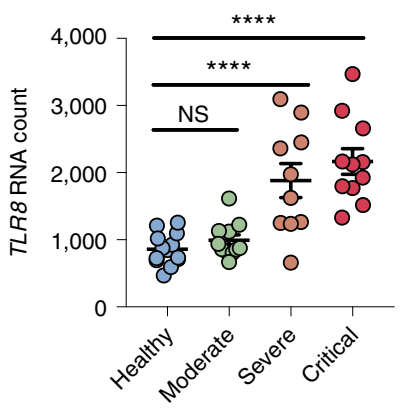

I

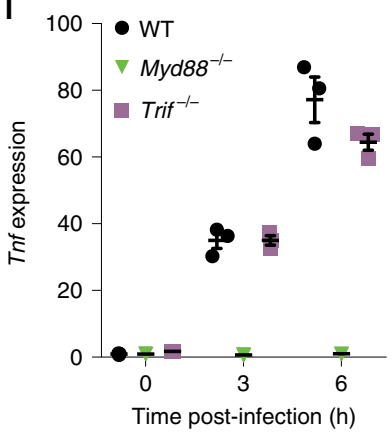

Time post-infection (h)

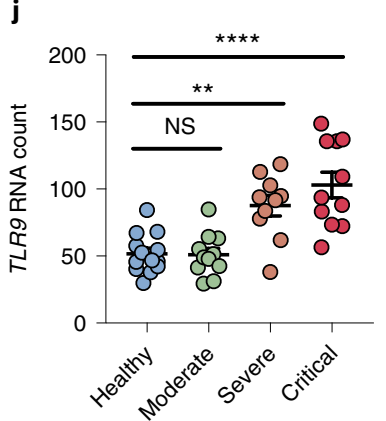

k

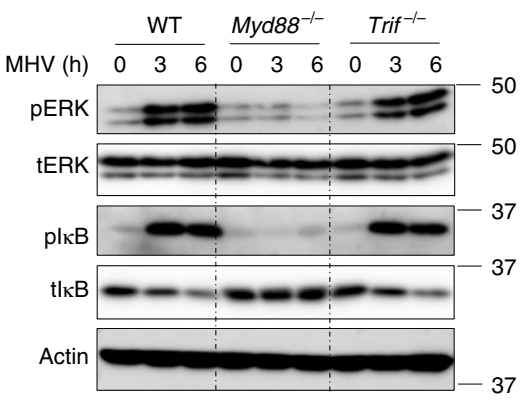

Fig. 1 Myd88 and TLRs are associated with the severity of COVID-19. a-j, Absolute RNA counts of MYD88 (a), TRIF (b), TLR1 (c), TLR2 (d), TLR3 (e), TLR4 (f), TLR5 (g), TLR7 (h), TLR8 (i) and TLR9 (j) in patients with mild-to-moderate $(n=11)$, severe $(n=10)$ and critical $(n=11)$ COVID-19, and 13 healthy

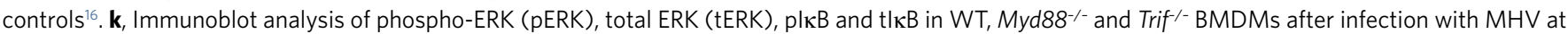
an $\mathrm{MOI}$ of 0.1 for the indicated time. Actin was used as the internal control. I, RT-PCR analysis of Tnf expression in WT, Myd88 $8^{-/-}$and Trif/- BMDMs after infection with $\mathrm{MHV}$ at an $\mathrm{MOI}$ of 0.1 for the indicated time, presented relative to levels of the host gene Gapdh. Significant differences compared with the healthy group are denoted as: ${ }^{\star} P<0.05,{ }^{\star \star} P<0.01,{ }^{\star \star \star} P<0.001$ and ${ }^{\star \star \star \star} P<0.0001$; NS, not significant (one-way ANOVA). Exact $P$ values are presented in Supplementary Table 1. Data are shown as mean \pm s.e.m. (a-j and $\mathbf{I})$. Data represent three independent experiments $(\mathbf{k}$ and $\mathbf{I})$.

in cytokine storm-induced mortality in patients with COVID-19 (ref. ${ }^{6}$ ). We found that TLR2 can sense the envelope protein from SARS-CoV-2 and was required for inflammatory cytokine release during $\beta$-coronavirus infection. Blocking TLR2 also protected against SARS-CoV-2-induced lethality in vivo. Our findings suggest an essential role of TLR2 in the disease development of COVID-19 and identify potential strategies for therapeutic intervention against this deadly pandemic disease. 


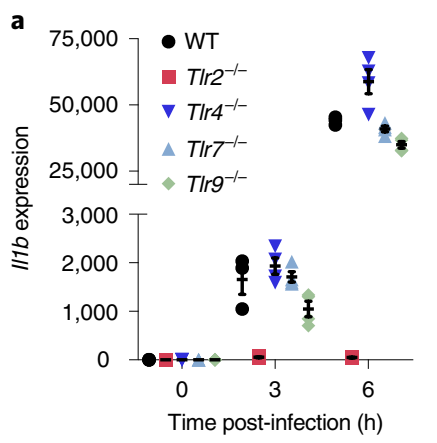

d

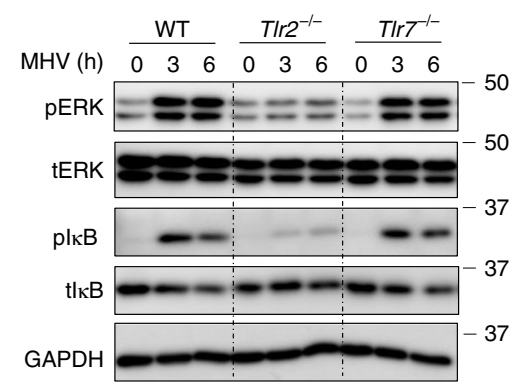

g

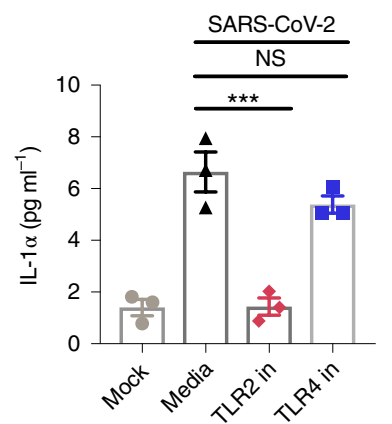

j

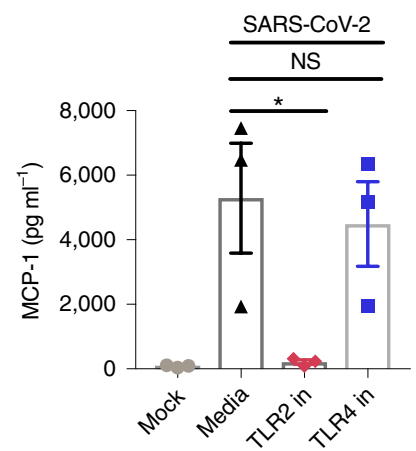

b

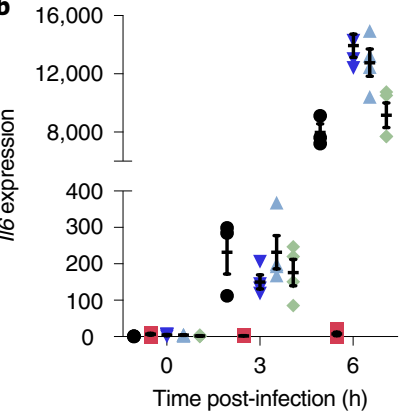

e

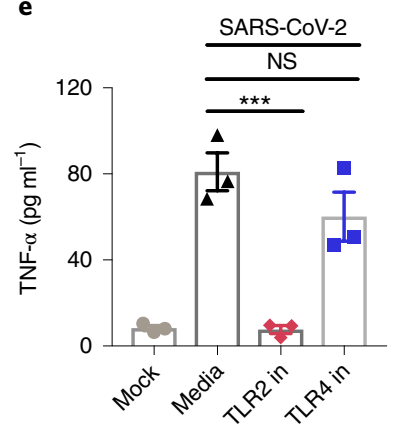

h

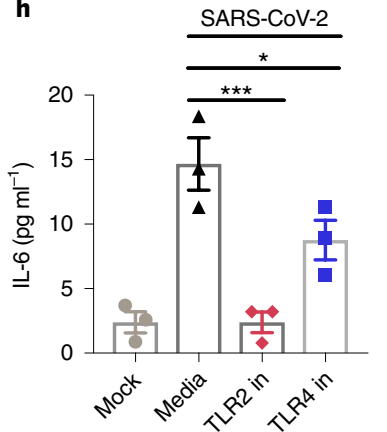

k

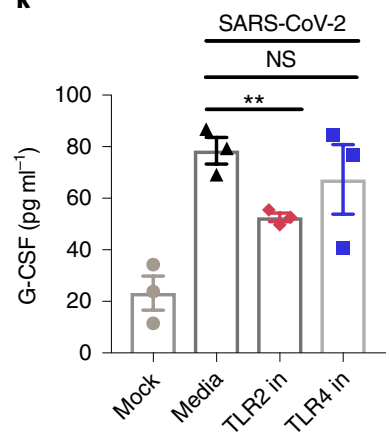

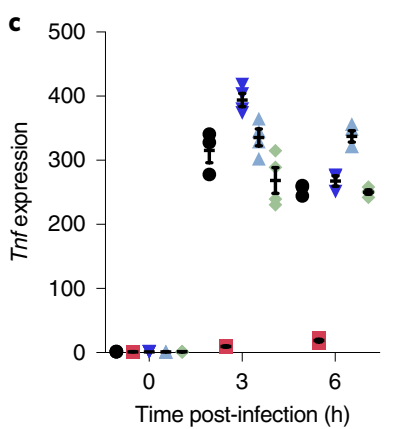

f
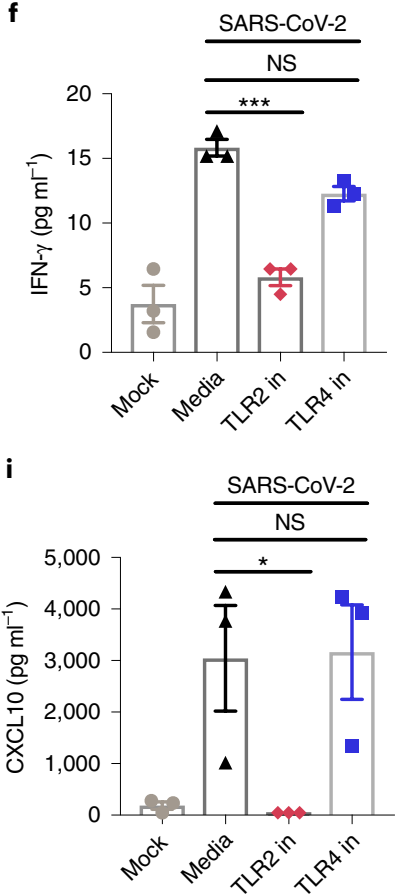

I

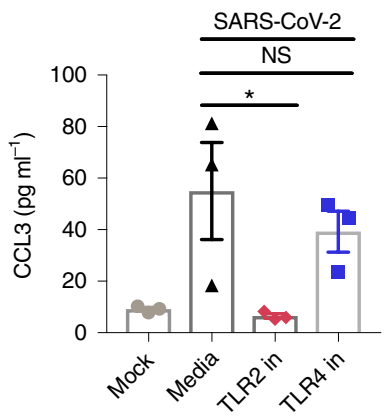

Fig. 2 | TLR2 is required for the inflammatory response during $\boldsymbol{\beta}$-coronavirus infection. a-c, RT-PCR analysis of the expression of $/ 17 b$ (a), I/6 (b) and Tnf

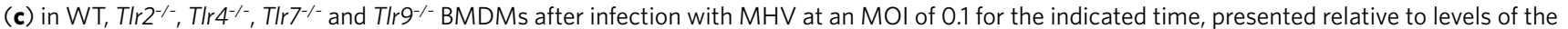

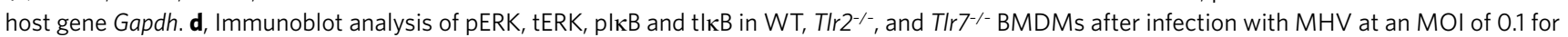
the indicated time. GAPDH was used as the internal control. e-I, TNF- $\alpha(\mathbf{e})$, IFN- $\gamma(\mathbf{f})$, IL-1 $\alpha(\mathbf{g})$, IL-6 (h), CXCL10 (i), MCP-1 (j), G-CSF (k) and CCL3 (I) release from human PBMCs infected with SARS-CoV-2 at an MOI of 0.5 for $20 \mathrm{~h}$ with or without the TLR2 inhibitor (oxPAPC; TLR2 in) or TLR4 inhibitor (CLI-095; TLR4 in). Significant differences compared with the media control infection group are denoted as: ${ }^{\star} P<0.05$ and ${ }^{\star \star \star} P<0.001 ;$ NS, not significant (one-way ANOVA) (e-I). Exact $P$ values are presented in Supplementary Table 1. Data represent three independent experiments (a-d) or two independent experiments $(\mathbf{e}-\mathbf{I})$. Data are shown as mean \pm s.e.m. $(n=3$ biological replicates; $\mathbf{a}-\mathbf{c}$ and $\mathbf{e}-\mathbf{I})$.

\section{Results}

Myd88 and TLRs are associated with the severity of COVID-19. Myd88 is important for proinflammatory cytokine production during $\mathrm{MHV}$ and SARS-CoV-1 infection ${ }^{14,15}$. To determine whether
Myd88 or another TLR adapter TRIF (TIR-domain-containing adapter-inducing interferon- $\beta$ ) plays a role in SARS-CoV-2-induced inflammatory responses and pathogenesis, we first reanalyzed a publicly available dataset ${ }^{16}$ for MYD88 and TRIF expression in patients 
a

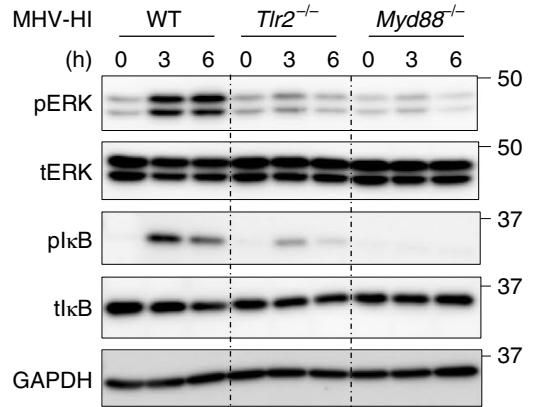

d

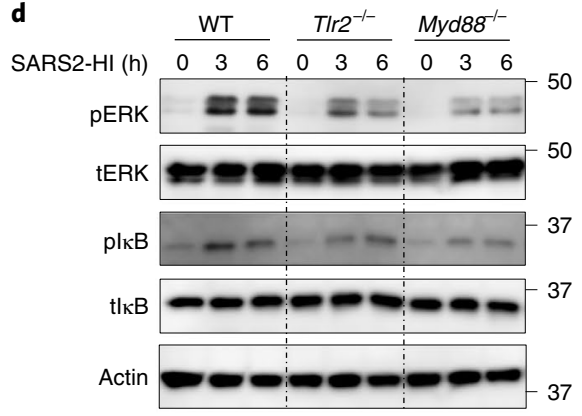

b

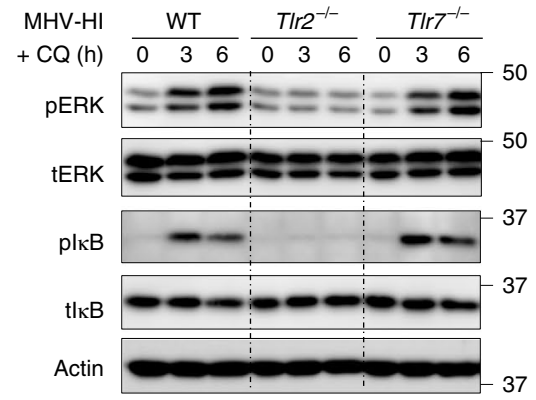

c

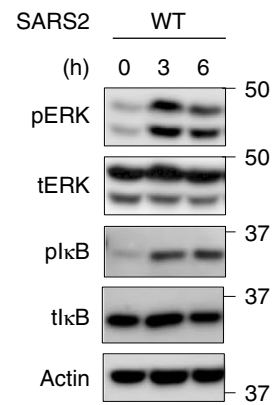

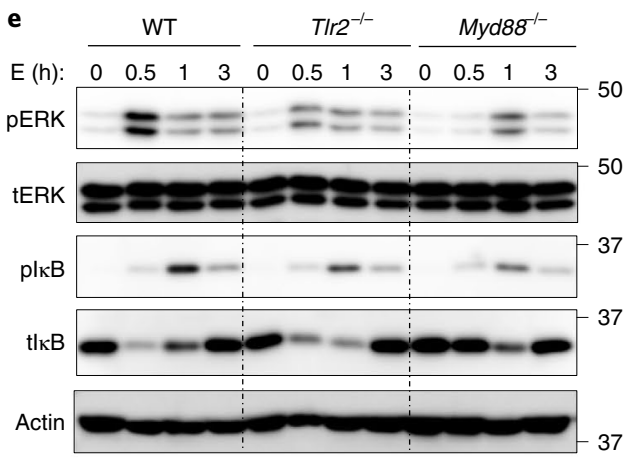

f

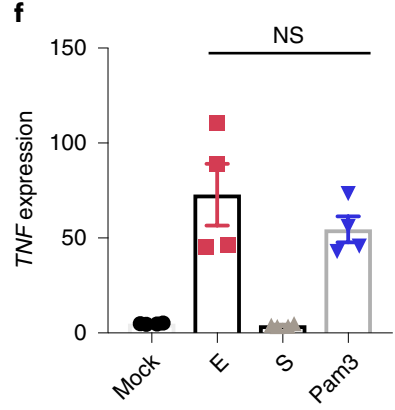

i

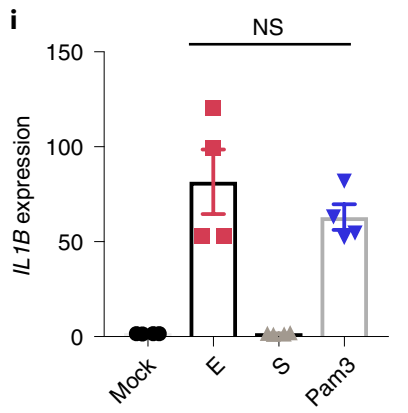

g

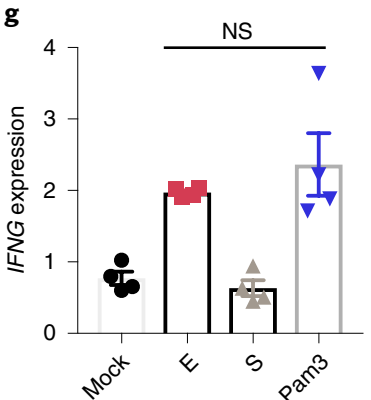

j

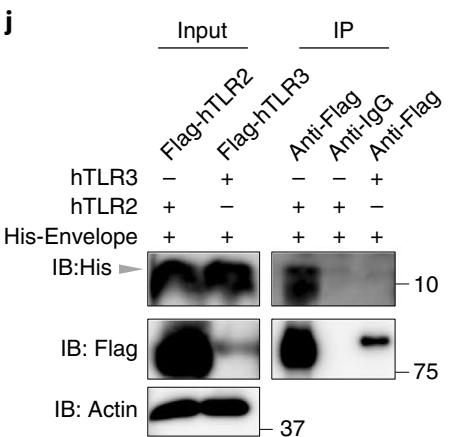

h

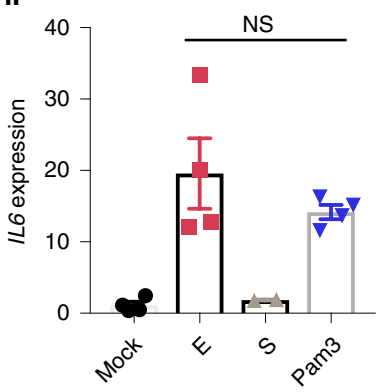

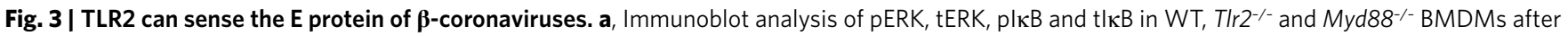
stimulation with $\mathrm{MHV}-\mathrm{HI}$ at an $\mathrm{MOI}$ of 0.1 for the indicated time. GAPDH was used as the internal control. $\mathbf{b}$, Immunoblot analysis of $\mathrm{pERK}$, tERK, plkB and $\mathrm{t} / \mathrm{KB}$ in $\mathrm{WT}$, T/r2 ${ }^{-/-}$and $T / r 7^{-1-}$ BMDMs after stimulation with $\mathrm{MHV}-\mathrm{HI}$ for the indicated time. Chloroquine (CQ) at a final concentration of $10 \mu \mathrm{M}$ was

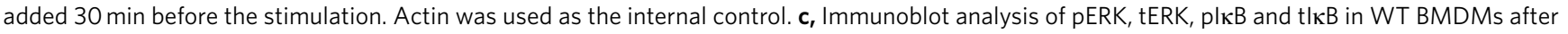
stimulation with SARS-CoV-2 (SARS2) for the indicated time. Actin was used as the internal control. d, Immunoblot analysis of $\mathrm{pERK}$, tERK, plkB and t/ $\mathrm{kB}$ in WT, T/r2 ${ }^{-/-}$and Myd88 $\%$ BMDMs after stimulation with SARS2-HI for the indicated time. Actin was used as the internal control. e, Immunoblot

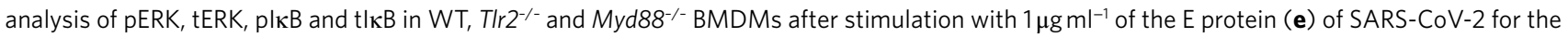
indicated time. Actin was used as the internal control. f-i, RT-PCR analysis of the expression of TNF (f), IFNG (g), IL6 (h) and IL1B (i) in human PBMCs after stimulation with $1 \mu \mathrm{g} \mathrm{ml}^{-1}$ of the E or S protein from SARS-CoV-2 or Pam3CSK4 (Pam3) for $4 \mathrm{~h}$, presented relative to levels of the host gene GAPDH. j, Immunoprecipitates and total lysates from 293T cells after incubation of purified E protein from SARS-CoV-2 with overexpressed human TLR2 or TLR3, or mouse TLR2. Student's $t$-test was used for statistical analysis between E protein- and Pam3-treated groups. IP, immunoprecipitation; NS, not significant. Data represent three independent experiments $(\mathbf{a}-\mathbf{j})$ (two-sided Student's $t$-test; $\mathbf{f}-\mathbf{i})$. Data are shown as mean \pm s.e.m. $(n=2$ biological replicates +2 technical replicates; $\mathbf{f}-\mathbf{i})$. 
with differing severities of COVID-19 (Fig. 1a,b). We found that MYD88 expression showed a positive correlation with the pathogenesis of COVID-19 (Fig. 1a), suggesting that MYD88 is associated with SARS-CoV-2 infection in humans. By contrast, TRIF was significantly elevated only in patients with critical COVID-19 (Fig. 1b). Myd88 is a key adapter shared by most TLRs. So far, ten TLRs have been identified in humans, named from TLR1 to TLR10 (ref. ${ }^{17}$ ). With the exception of TLR3, which signals exclusively through TRIF, all other TLRs utilize Myd88 to trigger inflammatory cytokine production ${ }^{17}$. To investigate whether any TLRs were positively correlated with COVID-19 severity, as Myd88 was, we reanalyzed the dataset ${ }^{16}$ for TLR expression in patients with differing severities of COVID-19 (Fig. $1 \mathrm{c}-\mathrm{j}$ ). We found that the expression pattern of TLR2 was increased with severity of COVID-19, similar to the phenomenon observed with MYD88 (Fig. 1d). Also, the expression of TLR1, TLR4, TLR5, TLR8 and TLR9 was significantly elevated in patients with severe and critical COVID-19 (Fig. 1c,f,g,i,j). By contrast, expression of TLR3 did not show any correlation with the disease development of COVID-19, and the expression of TLR7 was increased only in patients with moderate COVID-19 (Fig. 1e,h). All these data suggest an association of Myd88 and certain TLRs (TLR1, TLR2, TLR4, TLR5, TLR8 and TLR9) with disease progression in patients with COVID-19.

To confirm the role of Myd88 in $\beta$-coronavirus-induced inflammatory responses, we infected mouse bone marrow-derived macrophages (BMDMs) with MHV, the prototype of this genus that mimics many of the key aspects of human coronavirus biology and can be investigated under biosafety level 2 conditions ${ }^{18}$. Inflammatory cytokine expression largely depends on the extracellular signal-regulated kinase (ERK) and nuclear factor $\kappa$-light-chain-enhancer of activated B cell (NF- $\kappa \mathrm{B})$ signaling pathways ${ }^{19,20}$, and the NF- $\kappa \mathrm{B}$ pathway is activated in patients with COVID-19 (ref. ${ }^{16}$ ). In line with this, we found activation of NF- $\mathrm{BB}$ by MHV infection in BMDMs, as indicated by the phosphorylation

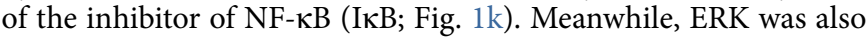
activated after MHV infection (Fig. 1k). Consistent with previous reports that Myd88 is required for $\beta$-coronavirus-induced inflammatory responses ${ }^{14,15}$, loss of Myd88 abolished the activation of both ERK and NF- $\mathrm{KB}$ after MHV infection (Fig. 1k). In contrast, TRIF was dispensable for MHV-induced ERK and NF- $\mathrm{KB}$ activation (Fig. 1k). In agreement with the activation of signaling pathways, transcription of inflammatory cytokine genes $T n f, I l 1 b$ and Il6 was induced after MHV infection in wild-type (WT) and Trif ${ }^{-1}$

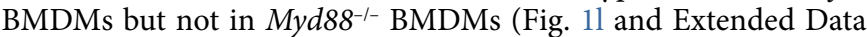
Fig. 1a,b). Given that the type I IFNs are critical for the development of COVID-19 (ref. ${ }^{16}$ ), we also assessed the role of the innate sensors required for the induction of type I IFNs after $\beta$-coronavirus infection using $\mathrm{Mda5}^{-1-}$ and $\mathrm{Mavs}^{-/}$BMDMs. Consistent with published reports ${ }^{11,21}$, MDA5 was required for $\beta$-coronavirus-induced type I IFN expression (Extended Data Fig. 2a,b). Collectively, these data indicate that Myd88 and some TLRs are correlated with the disease development of COVID-19, and that Myd88 is essential for $\beta$-coronavirus-induced inflammatory cytokine expression, whereas MDA5 is required for type I IFN expression.

TLR2 is required for inflammatory responses to $\beta$-coronavirus. TLR2, $-3,-4,-7,-8,-9$ and -10 have been reported to recognize viral infection under certain conditions ${ }^{22,23}$. TLR3 is a TRIF-dependent innate sensor ${ }^{23}$ and TLR10 has been shown to be nonfunctional in mice $^{24}$. In addition, TLR8 functions controversially in mice, compared with its role in humans ${ }^{25}$. To determine which TLR(s) are responsible for sensing $\beta$-coronavirus infection and signaling through Myd88, we infected BMDMs deficient in TLR2, -4, -7 or -9 with MHV, and monitored the transcription of inflammatory cytokine genes. We found that loss of TLR4, -7 or -9 had no effect on MHV-induced expression of $I l 1 b$, Il6 or Tnf in BMDMs, whereas TLR2 deficiency abolished transcription of each of these genes (Fig. $2 \mathrm{a}-\mathrm{c}$ ). This suggests that TLR2 is the innate sensor that triggers $\beta$-coronavirus-induced inflammatory cytokine expression. To further confirm the role of TLR2 during $\beta$-coronavirus infection, we examined the activation of inflammatory signaling pathways in $T l r 2^{-/-}$BMDMs after MHV infection. MHV-induced activation of ERK and NF- $\kappa$ B was abrogated in Tlr2 ${ }^{-1-}$ BMDMs (Fig. 2d). By contrast, TLR7 deficiency had no effect on MHV-induced activation of ERK and NF- $\kappa$ B (Fig. $2 d$ ). In addition, secretion of IL- 6 and TNF- $\alpha$ was abolished in Tlr2 ${ }^{-1-}$ BMDMs infected with MHV, whereas loss of TLR7 did not affect the release of these two cytokines (Extended Data Fig. 3a,b). Release of inflammatory chemokines, including CXCL10 (IP-10), CCL3 (macrophage inflammatory protein (MIP)-1 $\alpha$ ), CXCL1 (KC), RANTES (regulated upon activation, normal T cell expressed and presumably secreted), monocyte chemoattractant protein-1 (MCP-1) and granulocyte colony-stimulating factor (G-CSF), was also reduced or completely eliminated in $\mathrm{Tlr}^{-1-}$ BMDMs after MHV infection at 3 and $6 \mathrm{~h}$ post-infection, whereas loss of TLR7 had minimal effect on the expression of these chemokines (Extended Data Fig. 3c-h). These findings suggest that TLR2 specifically is required for the expression of these inflammatory cytokines and chemokines after MHV infection.

Studies on serum cytokines in patients with COVID-19 have indicated that many cytokines and chemokines, such as IL- 6 , TNF- $\alpha$, IFN- $\gamma$, IL-1 $\alpha$, IL-1 receptor antagonist (IL-1Ra), G-CSF, MCP-1, CCL3 and CXCL10, are elevated in response to SARS-CoV-2 infection and positively correlated with disease severity ${ }^{4,16,26}$. To investigate the role of TLR2 in SARS-CoV-2-induced cytokine and chemokine expression, we infected human peripheral blood mononuclear cells (PBMCs) with SARS-CoV-2 in the presence of a TLR2 inhibitor. First, we identified an optimal TLR2 inhibitor by confirming the effectiveness and specificity of two different inhibitors on TLR2 signaling in BMDMs stimulated with a TLR2 ligand, Pam3CSK4 (Pam3). We found that oxPAPC was more potent in inhibiting Pam3-induced inflammatory signaling than C29 (Extended Data Fig. 4a). In addition, oxPAPC had no effect on activation of ERK and NF- $\mathrm{KB}$ signaling induced by a TLR7 ligand, R848 (Extended Data Fig. 4b), suggesting that oxPAPC specifically inhibits TLR2-dependent inflammatory responses. In human PBMCs, the inhibition of TLR2 signaling by oxPAPC treatment significantly reduced SARS-CoV-2-induced secretion of TNF- $\alpha$, IFN- $\gamma$, IL- $1 \alpha$, IL-6, CXCL10, MCP-1, G-CSF and CCL3 (Fig. 2e-1). By contrast, the TLR4-specific inhibitor had minimal effects on the release of these cytokines and chemokines in response to SARS-CoV-2 infection (Fig. 2e-1).

Taken together, these results indicate that TLR2 can sense $\beta$-coronavirus infection and is responsible for $\beta$-coronavirus-induced inflammatory cytokine production.

TLR2 can sense the E protein of $\boldsymbol{\beta}$-coronaviruses. TLR2 is a cell surface innate immune sensor with the ability to recognize diverse ligands from viruses, fungi, bacteria and parasites ${ }^{27}$. TLR2 has been reported to sense several viral proteins after infection, including the dUTPase of Epstein-Barr virus, the glycoprotein B of cytomegalovirus and the capsid of hepatitis B virus ${ }^{27}$. We therefore hypothesized that TLR2 recognizes viral proteins from $\beta$-coronaviruses as well. To investigate whether viral proteins independent of functioning virions could activate TLR2, we used heat-inactivated MHV $(\mathrm{MHV}-\mathrm{HI})^{28}$. MHV-HI activated ERK and NF- $\kappa \mathrm{B}$ signaling pathways in WT BMDMs (Fig. 3a). In contrast, this activation was reduced or eliminated in $\mathrm{Tlr}^{-/-}$and $\mathrm{Myd} 88^{-/-}$BMDMs (Fig. 3a). These data suggest that activation of the TLR2 signaling pathway by $\beta$-coronaviruses does not require viral replication. It has been reported that chloroquine, when added before infection, can inhibit coronavirus entry ${ }^{29}$. We also observed that the cell fusion during live $\mathrm{MHV}$ infection was suppressed by chloroquine treatment, further suggesting that chloroquine inhibits viral entry (Extended Data 
a
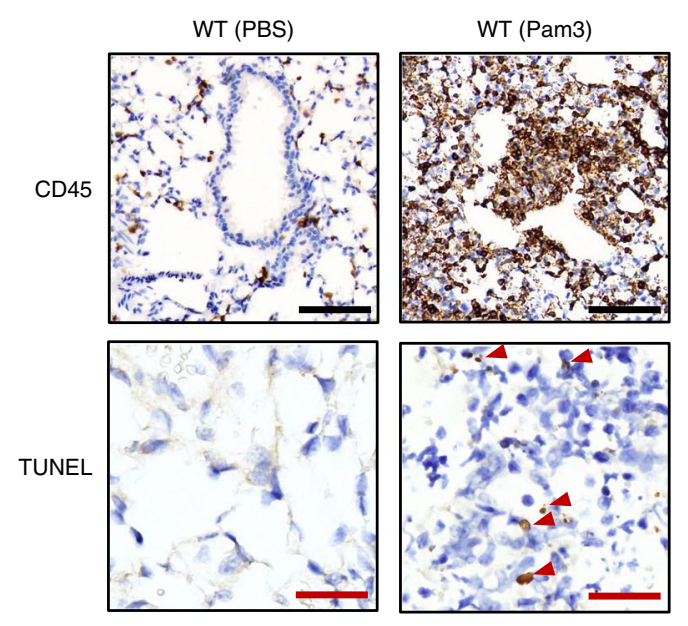
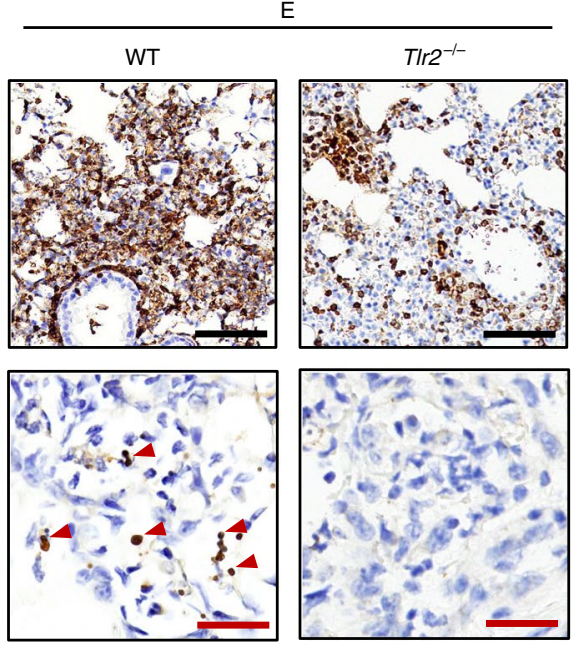

b
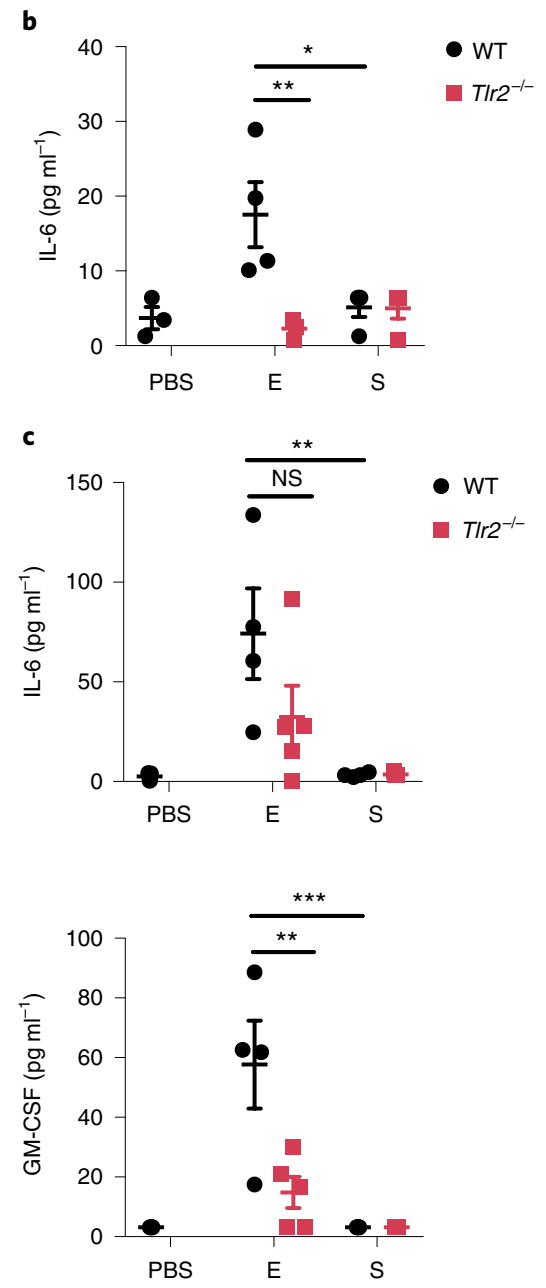
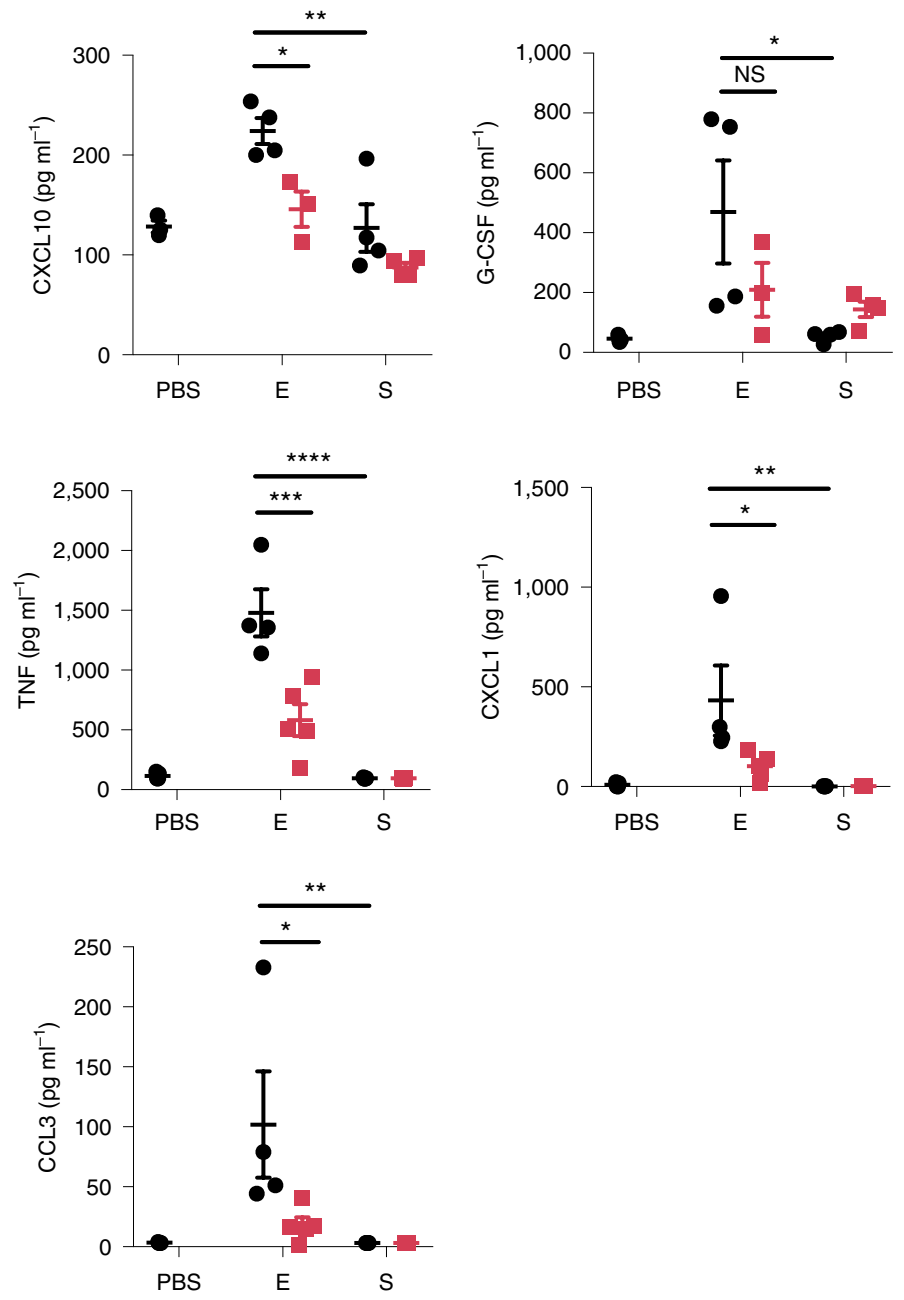

Fig. 4 | The E protein of SARS-CoV-2 can induce TLR2-dependent inflammation and damage in the lungs. a, CD45 immunostaining and TUNEL staining of lung samples obtained from mice $24 \mathrm{~h}$ after being intratracheally instilled with PBS ( $n=3$ biological replicates), the E protein from SARS-CoV-2 ( $n=4$ biological replicates) or Pam3CSK4 (Pam3; $n=4$ biological replicates). Red arrows indicate TUNEL-positive cells. Scale bar, $100 \mu \mathrm{m}$ (black) or $25 \mu \mathrm{m}$ (red). b, Levels of IL-6, CXCL10 and G-CSF in the serum of mice $24 \mathrm{~h}$ after being intratracheally instilled with PBS or the E or S protein from SARS-CoV-2. c, Levels of IL-6, TNF- $\alpha$, CXCL1, GM-CSF and CCL3 in the BALF of mice $6 \mathrm{~h}$ after being intratracheally instilled with PBS or the E or S protein from SARS-CoV-2. Significant differences compared with WT group treated with E protein are denoted as: ${ }^{\star} P<0.05,{ }^{\star \star} P<0.01$, ${ }^{\star \star \star} P<0.001$ and ${ }^{\star \star \star \star} P<0.0001$ (one-way ANOVA; $\mathbf{b}$ and $\mathbf{c})$. Exact $P$ values are presented in Supplementary Table 1. Data represent two independent experiments (a-c). Data are shown as mean \pm s.e.m. $(n=3-5$ biological replicates; $\mathbf{b}$ and $\mathbf{c})$. 
Fig. 5a). To test whether the activation of the TLR2 signaling pathway requires the internalization of viral proteins from $\beta$-coronaviruses, we treated BMDMs with $10 \mu \mathrm{M}$ chloroquine for $30 \mathrm{~min}$ before adding the MHV-HI. We observed that chloroquine treatment did not inhibit the activation of MHV-HI-induced TLR2-dependent inflammatory signaling pathways (Fig. 3b), suggesting that the ligands sensed by TLR2 in $\beta$-coronaviruses are viral surface proteins. Based on these findings, we hypothesized that, even though SARS-CoV-2 cannot infect murine cells due to the receptor specificity $^{30}$, it would still be able to activate the inflammatory signaling pathways in BMDMs. Indeed, SARS-CoV-2-stimulated BMDMs showed activation of the ERK and NF- $\kappa B$ signaling pathways (Fig. 3c). This may support the recent finding that SARS-CoV-2 inoculation induced lung inflammation in WT C57BL/6 mice ${ }^{31}$. To further confirm the ability of SARS-CoV-2 proteins to activate inflammatory signaling without infection, we used heat-inactivated SARS-CoV-2 (SARS2-HI) to stimulate the BMDMs. We found that SARS2-HI stimulated inflammatory signaling pathways, which were substantially reduced in TLR2- and Myd88-deficient BMDMs (Fig. 3d). Collectively, these data suggest that TLR2 can sense the surface proteins of $\beta$-coronaviruses before entry.

There are three viral proteins on the surface of $\beta$-coronaviruses, namely spike (S) protein, envelope (E) protein and membrane (M) protein $^{32}$. It was previously reported that a strain of SARS-CoV-1 lacking the $\mathrm{E}$ protein was not able to activate the NF- $\kappa \mathrm{B}$ pathway, thus significantly reducing the production of inflammatory cytokines after infection in mice ${ }^{33}$. To determine whether the $\mathrm{E}$ protein can be sensed by TLR 2 to activate inflammatory signaling pathways, we stimulated BMDMs with purified E protein from SARS-CoV-2. We found that the E protein activated ERK and NF- $\kappa B$ signaling pathways in WT BMDMs, and this activation was greatly attenuated

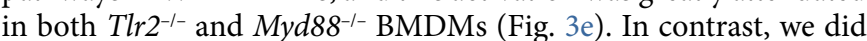
not observe any activation after stimulating BMDMs with the purified S protein from SARS-CoV-2 (Extended Data Fig. 6a). To further confirm the effect of the E protein on the activation of inflammatory signaling, we evaluated the expression of inflammatory cytokines in response to different doses of E protein. We found that the E protein could induce robust inflammatory cytokine expression at the concentration of $5 \mathrm{ng} \mathrm{ml}^{-1}$, although the effect was not as strong as the traditional TLR2 ligand Pam 3 at this concentration (Extended Data Fig. 6 b,c). When a higher concentration of E protein was added, its ability to induce inflammatory cytokine expression was comparable to that of Pam3 (Extended Data Fig. 6b,c). In addition, transcription of Illb, Il6 and Tnf was strongly upregulated in WT BMDMs stimulated with the E protein, but not with the $S$ protein, and the loss of TLR2 attenuated the expression of Il1b, Il6 and Tnf induced by the E protein (Extended Data Fig. 6d-f).

ERK and NF- $\kappa \mathrm{B}$ signaling is also required for transcriptional upregulation of Nlrp3 (ref. ${ }^{34}$ ), one of the prerequisite steps in NLRP3 inflammasome activation. To further confirm the effect of the E protein on activating the inflammatory signaling pathways in BMDMs, we evaluated Nlrp3 upregulation and ATP-induced NLRP3 inflammasome activation after priming with the E protein. We observed Nlrp3 upregulation and caspase-1 cleavage in WT BMDMs in response to priming with the E protein, whereas S-protein priming failed to induce the Nlrp3 upregulation and inflammasome activation (Extended Data Fig. 6g,h). The Nlrp3 upregulation and inflammasome activation

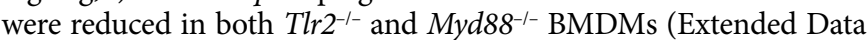
Fig. 6g,h). Consistently, pyroptosis activation and IL-18 release after E protein + ATP treatment were reduced in both Tlr $2^{-1-}$ and $\mathrm{Myd}^{-8^{-}}$

BMDMs, compared with those in WT BMDMs (Extended Data Fig. 6h,i). Together, these data indicate that the $\mathrm{E}$ protein of SARS-CoV-2 can activate TLR2-dependent signaling pathways.

To further examine the role of the E protein in driving inflammatory signaling in human cells, we stimulated human PBMCs with the E or S protein of SARS-CoV-2. We found that the E protein could induce expression of TNF, IFNG, IL6 and IL1B similar to that induced by Pam 3 in human PBMCs, whereas the $S$ protein could not induce the expression of these inflammatory cytokine genes (Fig. 3f-i). These data suggest that the E protein of $\beta$-coronaviruses can act as a PAMP in human cells. To verify whether TLR2 can directly interact with the E protein of SARS-CoV-2, we conducted co-immunoprecipitations with TLR2 and the E protein. We found that both human and mouse TLR2 could interact with the E protein of SARS-CoV-2 (Fig. 3j), further supporting the conclusion that the E protein of SARS-CoV-2 can induce inflammatory responses in both human and mouse cells.

Taken together, these data indicate that TLR2 can sense the E protein of $\beta$-coronaviruses to initiate inflammatory signaling pathways and cytokine production.

SARS-CoV-2 E protein induces TLR2-driven lung inflammation. Given that SARS-CoV-2 infection induces severe lung inflammation, we next determined whether the E protein could trigger lung inflammation in vivo. It has been reported that Pam 3 can induce lung inflammation after intratracheal instillation ${ }^{35}$. Consistent with this inflammation, we detected large numbers of $\mathrm{CD} 45^{+}$cells accumulating in the lungs of WT mice administered Pam3 (Fig. 4a). Administration of the E protein from SARS-CoV-2 also triggered the recruitment of large numbers of inflammatory cells in the lungs of WT mice, but not in the lungs of Tlr2 $2^{-/-}$mice (Fig. 4a). Also, there was no increase in $\mathrm{CD} 45^{+}$cells in the lungs of mice treated with the $\mathrm{S}$ protein compared with those administered phosphate-buffered saline (PBS; Extended Data Fig. 7a), which is consistent with our in vitro findings that the $\mathrm{S}$ protein does not induce inflammatory signaling. As overt inflammatory cytokine release can lead to tissue damage, we assessed whether administration of the E protein resulted in lung damage. We found that administration of Pam 3 triggered cell death in the mouse lungs (Fig. 4a). Similarly, E-protein administration also led to comparable cell death in the mouse lungs (Fig. 4a), suggesting that the E protein of SARS-CoV-2 has the potential to induce tissue damage during infection. By contrast, no cell death was detected in the lungs of mice treated with the $S$ protein (Extended Data Fig. 7a). Furthermore, we evaluated the concentrations of inflammatory cytokines and chemokines in the serum of the mice. We found that the levels of IL- 6 were elevated in WT mice after stimulating with the E protein of SARS-CoV-2 (Fig. 4b). By contrast, the amount of IL-6 in Tlr2 $2^{-/-}$mice administered the E protein or WT and Tlr2 $2^{-/-}$ mice administered the $S$ protein was close to the basal level, suggesting that there was no activation of IL- 6 production in these mice (Fig. 4b). Similarly, CXCL10 and G-CSF levels were increased in WT mice stimulated with the E protein but not in $T l r 2^{-1-}$ mice administered either the E or the S protein (Fig. 4b). To further confirm the inflammatory effect of the E protein in the lungs of mice, we collected the bronchoalveolar lavage fluid (BALF) at $6 \mathrm{~h}$ after intratracheal instillation. We found that the $S$ protein did not induce secretion of any of the cytokines tested in the BALF of both WT and $T l r 2^{-/-}$mice, whereas the E protein significantly increased the amounts of IL-6, TNF- $\alpha$, CXCL1, granulocyte-macrophage colony-stimulating factor (GM-CSF) and CCL3 in the BALF of WT mice (Fig. 4c). Taken together, these data indicate that the E protein of SARS-CoV-2 can induce TLR2-dependent inflammation in the lungs of mice.

TLR2 inhibitor protects against SARS-CoV-2 pathology in vivo. Given the critical role of TLR2 in SARS-CoV-2 infection-induced inflammatory cytokine release in vitro, we assessed whether blocking TLR2 signaling could affect the mortality of mice after infection with SARS-CoV-2. Due to the receptor specificity of SARS-CoV-2, we used mice expressing human angiotensin-converting enzyme 2 (ACE2) introduced under the control of the human cytokeratin 18 (K18), known as K18-hACE2 transgenic mice. Two doses of the TLR2 inhibitor were administered during the infection. Treating the 

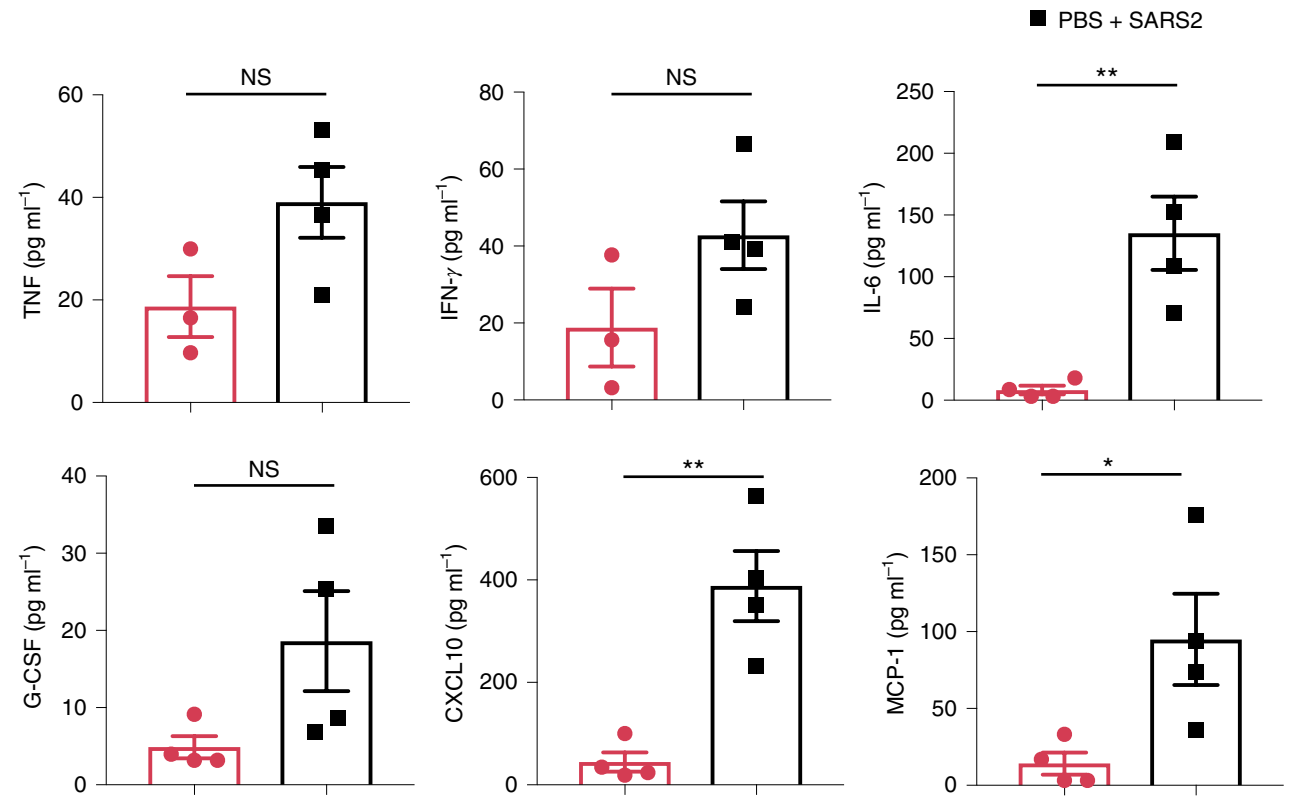

b

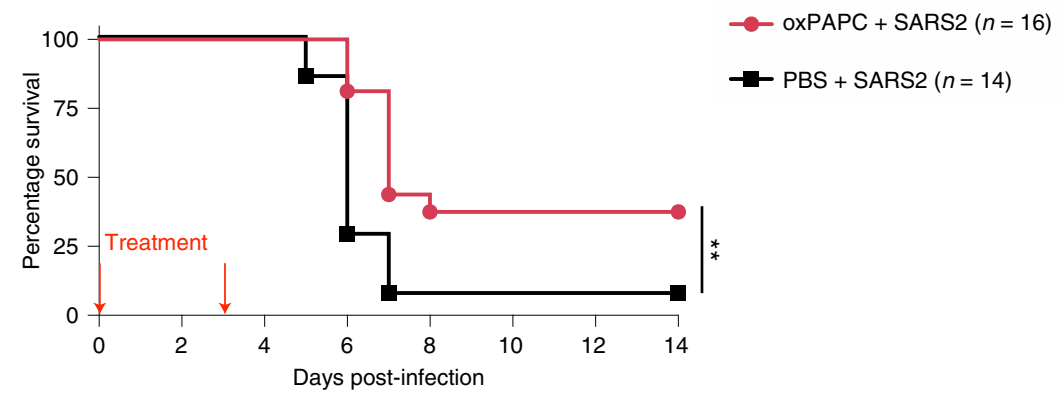

c

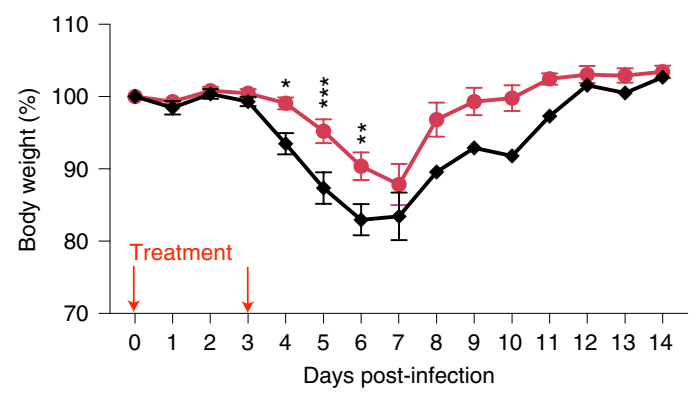

Fig. 5 | Blocking TLR2 signaling protects against SARS-CoV-2 infection in vivo. a, Levels of TNF- $\alpha$, IFN- $\gamma$, IL-6, G-CSF, CXCL10 and MCP-1 in the BALF of mice $2 \mathrm{~d}$ after SARS-CoV-2 infection. b, Pooled survival of 8-to-10-week-old K18-hACE2 transgenic mice after infection with SARS-CoV-2 ( $2 \times 10^{4}$ p.f.u. per mouse). $\mathbf{c}$, Pooled body weight change of infected mice in $\mathbf{b}$. Significant differences compared with the group treated with oxPAPC are denoted as: ${ }^{\star} P<0.05,{ }^{\star \star} P<0.01$, and ${ }^{\star \star \star} P<0.001$ (two-sided Student's $t$-test (a), log-rank test (b) or two-way ANOVA (c)). Exact $P$ values are presented in Supplementary Table 1. Data are shown as mean \pm s.e.m. ( $n=3-4$ biological replicates (a) or $n=14-16$ biological replicates (b and $\mathbf{c})$ ).

mice with the TLR2 inhibitor significantly reduced the release of IL-6, CXCL10 and MCP-1, and led to a nonsignificant decrease in the amounts of TNF- $\alpha$, IFN- $\gamma$ and G-CSF released in the BALF at day 2 post-infection (Fig. 5a). In addition, TLR2 inhibitor treatment led to significantly increased survival of the SARS-CoV-2-infected mice, compared with the control mice injected with PBS (Fig. 5b). In line with this, the body weight of mice treated with the TLR2 inhibitor decreased more slowly and recovered faster compared with the body weight change in the control group (Fig. 5c). Collectively, these data suggest that TLR2 senses SARS-CoV-2 infection in vivo and that blocking TLR2 signaling could provide protection against SARS-CoV-2 mortality.

\section{Discussion}

Inflammatory signaling leading to increases in cytokine secretion and cytokine storm are common phenotypes after $\beta$-coronavirus-induced zoonotic infection ${ }^{3,7}$. Recently, it has been shown that the inflammatory cytokines TNF- $\alpha$ and IFN- $\gamma$ specifically play a critical role in the pathogenesis of COVID-19 (ref. ${ }^{6}$ ). Although it is well known that $\beta$-coronavirus infections can trigger robust inflammatory cytokine release, the innate sensors that are required for inflammatory cytokine expression in this context have remained unclear. In the present study, we provided the first genetic evidence that TLR2 can sense $\beta$-coronavirus infection via recognition of the E protein to induce release of inflammatory cytokines, 
including TNF- $\alpha$ and IFN- $\gamma$. We also showed that the activation of TLR2 signaling in response to $\beta$-coronavirus infection was independent of viral entry and replication.

TLR2 is involved in sensing different kinds of pathogens, including bacteria, viruses, fungi and parasites ${ }^{27}$. The ligand diversity of TLR2 could be explained by its ability to form heterodimers with TLR1 or TLR6 (ref. ${ }^{27}$ ). Also, TLR10 can interact with TLR2 to sense some ligands under certain conditions ${ }^{36}$. The residual NF- $\mathrm{KB}$ and ERK signaling activation in Tlr2 $2^{-/-}$BMDMs stimulated with the E protein of SARS-CoV-2 could be due to recognition of the E protein by TLR1 or TLR6 to activate the inflammatory response. It is also possible that some other innate sensors may recognize the $\mathrm{E}$ protein. More studies are required to confirm whether this occurs.

The E protein is a structural protein of coronaviruses that has ion channel activity ${ }^{37}$. It can form protein-lipid pores in the membrane to allow ion transport, which could provide the activation signal for NLRP3 inflammasome assembly ${ }^{37}$. Therefore, the E protein may be able to provide both the priming and the activation signal for the NLRP3 inflammasome. It is probable that priming and then transfecting with the E protein would activate the NLRP3 inflammasome and lead to the processing of the pyroptosis executioner gasdermin $\mathrm{D}$ that we observed. The phenomenon is similar to LPS, the cell wall component of Gram-negative bacteria, which can trigger inflammatory responses and also activate pyroptosis. Therefore, the E protein of $\beta$-coronaviruses could be a virulence factor like LPS that can induce an endotoxic-like shock on its own in vivo. However, to test this hypothesis requires large amounts of purified E protein.

The effect of a cytokine storm on the development of COVID19 has been extensively discussed since SARS-CoV-2 emerged, and increases in serum levels of proinflammatory cytokines have been linked to disease pathogenesis and mortality ${ }^{8}$. Based on this evidence, several clinical trials are ongoing to block various cytokines, including IL-6 and TNF- $\alpha^{38}$. However, blocking IL-6 has had limited clinical success, and more recent work suggests that TNF- $\alpha$ and IFN- $\gamma$ are the two key cytokines to target to inhibit disease pathogenesis ${ }^{6}$. Therefore, identifying the sensor and ligand upstream of TNF- $\alpha$ and IFN- $\gamma$ production in response to SARS-CoV-2 infection may provide critical information for clinical trials and direct the use of drugs to target specific pathways. Our findings identify more targets that could be pursued for therapeutic strategies to prevent or treat COVID-19. TLR2 inhibitors or antibodies could be useful to suppress the production of inflammatory cytokines and chemokines, thereby preventing the onset of cytokine storm. In addition, targeting TLR2 would have fewer side effects than directly targeting Myd88, because Myd88 is also the adapter for other TLRs and IL-1R and IL-18R ${ }^{39}$; inhibition of Myd88 would lead to the suppression of other critical cellular responses. In the present study, inhibition of TLR2 signaling reduced the release of TNF- $\alpha$ and IFN- $\gamma$, but this reduction was not significant at the time point analyzed in the lungs of mice during SARS-CoV-2 infection. One possible explanation is that some other innate sensors could also regulate the expression of TNF- $\alpha$ and IFN- $\gamma$ in vivo, allowing their expression to potentially be restored or not significantly changed after TLR2 inhibitor administration. Further studies are required to clarify this. In addition, to treat COVID-19, doses and timing to block TLR2 signaling should be seriously investigated, because optimal proinflammatory cytokine levels are generally protective to the host during infections ${ }^{40}$.

Beyond the potential of blocking TLR2, antibodies targeting the E protein of SARS-CoV-2 could possess dual roles, neutralizing the viral particles and simultaneously preventing the sensing by TLR2 and reducing inflammatory cytokine production. However, the timing of these interventions in the inflammatory signaling pathways should be carefully evaluated. Broad and long-lasting blockade of cytokine production may lead to delayed viral clearance and tissue repair. Detailed studies are required to analyze the effects of cytokine inhibition in different infection phases on the development of COVID-19.

Overall, our study identified the key inflammatory signaling pathways activated during $\beta$-coronavirus infection, which greatly improves our understanding of the molecular mechanism of $\beta$-coronavirus-induced cytokine production. These findings provide critical insights into strategies that can be pursued to control the development of the ongoing COVID-19 pandemic.

\section{Online content}

Any methods, additional references, Nature Research reporting summaries, source data, extended data, supplementary information, acknowledgements, peer review information; details of author contributions and competing interests; and statements of data and code availability are available at https://doi.org/10.1038/ s41590-021-00937-x.

Received: 22 December 2020; Accepted: 16 April 2021; Published online: 7 May 2021

\section{References}

1. MacLachlan, N. J. \& Dubovi, E. J. (eds) in Fenner's Veterinary Virology Ch. 24 (Academic Press, 2017); https://doi.org/10.1016/B978-0-12-800946-8.00024-6

2. Hu, B., Guo, H., Zhou, P. \& Shi, Z.-L. Characteristics of SARS-CoV-2 and COVID-19. Nat. Rev. Microbiol. https://doi.org/10.1038/s41579-020-00459-7 (2020).

3. Channappanavar, R. \& Perlman, S. Pathogenic human coronavirus infections: causes and consequences of cytokine storm and immunopathology. Semin. Immunopathol. 39, 529-539 (2017).

4. Lucas, C. et al. Longitudinal analyses reveal immunological misfiring in severe COVID-19. Nature 584, 463-469 (2020).

5. Zheng, M. et al. Impaired NLRP3 inflammasome activation/pyroptosis leads to robust inflammatory cell death via caspase-8/RIPK3 during coronavirus infection. J. Biol. Chem. https://doi.org/10.1074/jbc.RA120.015036 (2020)

6. Karki, R. et al. Synergism of TNF- $\alpha$ and IFN- $\gamma$ triggers inflammatory cell death, tissue damage, and mortality in SARS-CoV-2 infection and cytokine shock syndromes. Cell https://doi.org/10.1016/j.cell.2020.11.025 (2020).

7. Jose, R. J. \& Manuel, A. COVID-19 cytokine storm: the interplay between inflammation and coagulation. Lancet Respir. Med. 8, e46-e47 (2020).

8. Del Valle, D. M. et al. An inflammatory cytokine signature predicts COVID-19 severity and survival. Nat. Med. 26, 1636-1643 (2020).

9. Amarante-Mendes, G. P. et al. Pattern recognition receptors and the host cell death molecular machinery. Front. Immunol. 9, 764 (2018).

10. Briard, B., Place, D. E. \& Kanneganti, T.-D. DNA sensing in the innate immune response. Physiology 35, 112-124 (2020).

11. Zalinger, Z. B., Elliott, R., Rose, K. M. \& Weiss, S. R. MDA5 is critical to host defense during infection with murine coronavirus. J. Virol. 89, 12330-12340 (2015).

12. Cervantes-Barragan, L. et al. Control of coronavirus infection through plasmacytoid dendritic-cell-derived type I interferon. Blood 109, 1131-1137 (2007)

13. Channappanavar, R. et al. IFN-I response timing relative to virus replication determines MERS coronavirus infection outcomes. J. Clin. Invest. 130, 3625-3639 (2019).

14. Sheahan, T. et al. MyD88 is required for protection from lethal infection with a mouse-adapted SARS-CoV. PLoS Pathog. 4, e1000240 (2008).

15. Zhou, H., Zhao, J. \& Perlman, S. Autocrine interferon priming in macrophages but not dendritic cells results in enhanced cytokine and chemokine production after coronavirus infection. Mbio 1, e00219-10 (2010).

16. Hadjadj, J. et al. Impaired type I interferon activity and inflammatory responses in severe COVID-19 patients. Science 369, 718-724 (2020).

17. El-Zayat, S. R., Sibaii, H. \& Mannaa, F. A. Toll-like receptors activation, signaling, and targeting: an overview. Bull. Natl Res. Cent. 43, 187 (2019).

18. Ghosh, S. et al. $\beta$-Coronaviruses use lysosomes for egress instead of the biosynthetic secretory pathway. Cell https://doi.org/10.1016/j.cell.2020.10.039 (2020)

19. Carter, A. B., Monick, M. M. \& Hunninghake, G. W. Both Erk and p38 kinases are necessary for cytokine gene transcription. Am. J. Respir. Cell Mol. Biol. 20, 751-758 (1999).

20. Liu, T., Zhang, L., Joo, D. \& Sun, S.-C. NF-кB signaling in inflammation. Signal Transduct. Target. Ther. 2, 17023 (2017).

21. Yang, D., Geng, T., Harrison, A. G. \& Wang, P. Differential roles of RIG-I-like receptors in SARS-CoV-2 infection. Preprint at bioRxiv https://doi. org/10.1101/2021.02.10.430677 (2021). 
22. Lee, S. M. Y. et al. Toll-like receptor 10 is involved in induction of innate immune responses to influenza virus infection. Proc. Natl Acad. Sci. USA 111, 3793-3798 (2014).

23. Lester, S. N. \& Li, K. Toll-like receptors in antiviral innate immunity. J. Mol. Biol. 426, 1246-1264 (2014).

24. Hasan, U. et al. Human TLR10 is a functional receptor, expressed by B cells and plasmacytoid dendritic cells, which activates gene transcription through MyD88. J. Immunol. 174, 2942-2950 (2005).

25. Barrat, F. J. TLR8: no gain, no pain. J. Exp. Med. 215, 2964-2966 (2018).

26. Huang, C. et al. Clinical features of patients infected with 2019 novel coronavirus in Wuhan, China. Lancet 395, 497-506 (2020).

27. Oliveira-Nascimento, L., Massari, P. \& Wetzler, L. M. The role of TLR2 in infection and immunity. Front. Immunol. 3, 79 (2012).

28. Kampf, G., Voss, A. \& Scheithauer, S. Inactivation of coronaviruses by heat. J. Hosp. Infect. https://doi.org/10.1016/j.jhin.2020.03.025 (2020).

29. Burkard, C. et al. Coronavirus cell entry occurs through the endo-/lysosomal pathway in a proteolysis-dependent manner. PLoS Pathog. 10, e1004502 (2014).

30. Hoffmann, M. et al. SARS-CoV-2 cell entry depends on ACE2 and TMPRSS2 and is blocked by a clinically proven protease inhibitor. Cell 181, 271-280.e8 (2020).

31. Oladunni, F. S. et al. Lethality of SARS-CoV-2 infection in K18 human angiotensin-converting enzyme 2 transgenic mice. Nat. Commun. 11, 6122 (2020).

32. Siu, Y. L. et al. The M, E, and N structural proteins of the severe acute respiratory syndrome coronavirus are required for efficient assembly, trafficking, and release of virus-like particles. J. Virol. 82, 11318-11330 (2008).
33. DeDiego, M. L. et al. Inhibition of NF- $\mathrm{KB}$-mediated inflammation in severe acute respiratory syndrome coronavirus-infected mice increases survival. J. Virol. 88, 913-924 (2014).

34. Zhao, W., Ma, L., Cai, C. \& Gong, X. Caffeine inhibits NLRP3 inflammasome activation by suppressing MAPK/NF- $\kappa \mathrm{B}$ and A2aR signaling in LPS-induced THP-1 macrophages. Int. J. Biol. Sci. 15, 1571-1581 (2019).

35. Cabanski, M. et al. Genome-wide transcriptional profiling of mononuclear phagocytes recruited to mouse lungs in response to alveolar challenge with the TLR2 agonist Pam3CSK4. Am. J. Physiol. Lung Cell. Mol. Physiol. 297, L608-L618 (2009).

36. Guan, Y. et al. Human TLRs 10 and 1 share common mechanisms of innate immune sensing but not signaling. J. Immunol. 184, 5094-5103 (2010).

37. Nieto-Torres, J. L. et al. Severe acute respiratory syndrome coronavirus E protein transports calcium ions and activates the NLRP3 inflammasome. Virology 485, 330-339 (2015).

38. Lythgoe, M. P. \& Middleton, P. Ongoing clinical trials for the management of the COVID-19 pandemic. Trends Pharmacol. Sci. 41, 363-382 (2020).

39. Loiarro, M., Ruggiero, V. \& Sette, C. Targeting TLR/IL-1R signalling in human diseases. Mediators Inflamm. 2010, e674363 (2010).

40. Slaats, J., ten Oever, J., van de Veerdonk, F. L. \& Netea, M. G. IL-1 $\beta /$ IL-6/CRP and IL-18/ferritin: distinct inflammatory programs in infections. PLoS Pathog. 12, e1005973 (2016)

Publisher's note Springer Nature remains neutral with regard to jurisdictional claims in published maps and institutional affiliations.

(C) The Author(s), under exclusive licence to Springer Nature America, Inc. 2021 


\section{Methods}

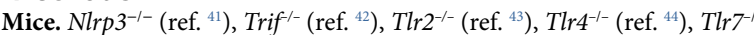

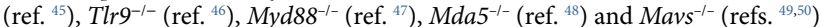
mice have been described previously. For SARS-CoV-2 infections, K18-ACE-2 transgenic mice were purchased from the Jackson Laboratory (stock no. 034860). All mice were bred at the Animal Resources Center at St Jude Children's Research Hospital and were backcrossed to the C57BL/6 background. Mice were kept with a 12:12 light:dark cycle. Humidity was maintained between $30 \%$ and $70 \%$ and the temperature ranged from $20^{\circ} \mathrm{C}$ to $23.3^{\circ} \mathrm{C}$. Age- and sex-matched, 6- to 9-week-old male and female mice were used for intratracheal instillation and 8- to 10-week-old male mice were used for in vivo SARS-CoV-2 infection studies. Noninfectious animal studies were conducted under protocols approved by the St Jude Children's Research Hospital Committee on the Use and Care of Animals. SARS-CoV-2 infections were performed at the University of Tennessee Health Science Center under animal biological safety level 3 conditions in accordance with the approval of the Institutional Animal Care and Use Committee of University of Tennessee Health Science Center (protocol no. 20-0132).

BMDMs. Primary BMDMs were cultured for $6 \mathrm{~d}$ in Iscove's modified Dulbecco's medium (Thermo Fisher Scientific, catalog no. 12440-053) supplemented with $10 \%$ fetal bovine serum (FBS; Biowest, catalog no. S1620), 30\% L929-conditioned medium, $1 \%$ nonessential amino acids (Thermo Fisher Scientific, catalog no. 11140-050) and 1\% penicillin-streptomycin (Thermo Fisher Scientific, catalog no. 15070-063). Then BMDMs were seeded into 12-well plates at a density of 1 million cells per well and incubated overnight before use.

MHV propagation. The MHV (A59 strain) was amplified in 17Cl-1 cells as previously described ${ }^{51}$. Briefly, $17 \mathrm{Cl}-1$ cells were inoculated with MHV-A59 at a multiplicity of infection (MOI) of 0.1 . At $48 \mathrm{~h}$ post-infection, the whole flask with medium and cells was frozen at $-80^{\circ} \mathrm{C}$ and then thawed at $37^{\circ} \mathrm{C}$. After repeating the freezing-thawing cycle twice, the supernatant was collected and centrifuged at 2,000 $\mathrm{g}$ for $10 \mathrm{~min}$ to remove the cell debris. Then the virus was purified by ultracentrifugation at 30,000 r.p.m. for $1 \mathrm{~h}$, after which the pellets were resuspended with fresh medium. The virus titer was measured by plaque assay in $17 \mathrm{Cl}-1$ cells.

Isolation of PBMCs. Whole blood was obtained from anonymous healthy donors who provided informed consent at St Jude Children's Research Hospital following institution review board-approved protocols. PBMCs were harvested from the blood by density gradient using Percoll (GE Healthcare, catalog no. 17-5445-01). PBMCs were maintained in RPMI-1640 supplemented with 10\% FBS.

SARS-CoV-2 culture. The SARS-CoV-2 isolate USA-WA1/2020 was obtained from BEI Resources (National Institute of Allergy and Infectious Diseases (NIAID), National Institutes of Health (NIH): SARS-Related Coronavirus 2, Isolate USA-WA1/2020, catalog no. NR-52281) and propagated in Vero-E6 cells (American Type Culture Collection (ATCC), catalog no. VERO C1008) at an MOI of 0.1 in minimal essential medium (Corning, catalog no. 17-305-CV) supplemented with 5\% heat-inactivated FBS (Gibco), 1\% L-glutamine (Corning, catalog no. 25-005-Cl) and $5 \mathrm{mM}$ penicillin-streptomycin (Gibco, catalog no. 30-001-Cl). The viral titer was measured by plaque assay as described previously for alphaviruses ${ }^{52}$. All experiments with live SARS-CoV-2 were conducted in a biosafety level 3 laboratory.

Cell stimulation/infection. For chemical ligand-induced inflammatory signaling responses, $1 \times 10^{6} \mathrm{BMDMs}$ seeded in 12 -well plates were pretreated with oxPAPC (Invivogen, tlrl-oxp1) at a final concentration of $30 \mathrm{\mu g} \mathrm{ml}^{-1}$ or C29 (MedChem Express, catalog no. HY-100461) at a final concentration of $50 \mu \mathrm{M}$ for $1 \mathrm{~h}$. Then the cells were stimulated with $1 \mu \mathrm{g} \mathrm{ml}^{-1}$ of Pam3CSK4 (Pam3) or $1 \mu \mathrm{g} \mathrm{ml}^{-1}$ of R848 for the indicated time. To check the NLRP3 inflammasome activation after viral protein priming, $1 \times 10^{6} \mathrm{BMDMs}$ seeded in 12 -well plates were stimulated with the E protein (ABclonal Technology, catalog no. RP01263) or S protein (ABclonal Technology, catalog no. RP01283LQ) of SARS-CoV-2 at a final concentration of $1 \mu \mathrm{g} \mathrm{ml}^{-1}$ for $4 \mathrm{~h}$ and then treated for $45 \mathrm{~min}$ with $5 \mathrm{mM}$ ATP (Roche, catalog no. 10127531001).

For MHV infection, $1 \times 10^{6} \mathrm{BMDMs}$ seeded in 12 -well plates were infected at an MOI of 0.1 in Dulbecco's modified Eagle's medium (Sigma, catalog no. D6171). After $2 \mathrm{~h}$ of incubation, cells were supplemented with $10 \%$ FBS. Samples were collected at the indicated timepoints. For MHV-HI stimulation, MHV was inactivated at $65^{\circ} \mathrm{C}$ for $30 \mathrm{~min}$, and then inactivated $\mathrm{MHV}$ at a volume equivalent to an MOI of 0.1 of live MHV was added into the BMDMs for the indicated time.

For SARS-CoV-2 infection of BMDMs, $1 \times 10^{6} \mathrm{BMDMs}$ were seeded into 12 -well plates and infected at an MOI of 0.5 for the indicated time. Cells were lysed with radioimmunoprecipitation assay lysis buffer. For PBMC infection, $1 \times 10^{6}$ PBMCs from 3 healthy donors were seeded into 24 -well plates and infected at an MOI of 0.5. Supernatants from mock-treated and infected PBMCs were collected after infection for $20 \mathrm{~h}$. To inhibit TLR2 activity, oxPAPC was used at a final concentration of $30 \mathrm{\mu g} \mathrm{ml}^{-1}$ together with SARS-CoV-2. To inhibit TLR4 activity, CLI-095 was used at a final concentration of $1 \mu \mathrm{g} \mathrm{ml}^{-1}$ together with SARS-CoV-2. The heat-inactivated SARS-CoV-2 was obtained through BEI
Resources (NIAID, NIH: SARS-Related Coronavirus 2, Isolate USA-WA1/2020, Heat Inactivated, NR-52286)

Cell fusion analysis. BMDMs $\left(10^{6}\right.$ cells per well) seeded into 12 -well plates were treated with $10 \mu \mathrm{M}$ chloroquine for $30 \mathrm{~min}$. Then the cells were infected with MHV at an MOI of 0.1. At $8 \mathrm{~h}$ post-infection, DNA stain, the NUCLEAR-ID Red (Enzo, catalog no. ENZ-52406), was added to the cells after diluting 5,000-fold. Images were analyzed using IncuCyte S3 software.

Co-immunoprecipitation assay. Plasmids expressing Flag-tagged human TLR2, TLR3 or mouse TLR2 were transfected into 293T (ATCC, catalog no.CRL-3216) cells for $48 \mathrm{~h}$, then cells were lysed in NP-40 lysis buffer ( $0.1 \% \mathrm{NP}-40,150 \mathrm{mM}$ $\mathrm{NaCl}, 50 \mathrm{mM}$ 4-(2-hydroxyethyl)-1-piperazine-ethanesulfonic acid), and $20 \mathrm{~min}$ later cell lysates were centrifuged at 13,000 r.p.m. for $10 \mathrm{~min}$. Supernatant was collected and incubated with $5 \mu \mathrm{g}$ of purified E protein of SARS-CoV-2 and $1.5 \mu \mathrm{g}$ of the indicated primary antibody on a rocking platform at $4^{\circ} \mathrm{C}$. After overnight incubation, protein A/G PLUS-Agarose (Santa Cruz Biotechnology) beads were added. After $2 \mathrm{~h}$, the beads were collected by centrifugation after washing with lysis buffer $4 \times$. Finally, samples were harvested after boiling in $2 \times$ sodium dodecylsulfate loading buffer at $100^{\circ} \mathrm{C}$ for $5 \mathrm{~min}$.

SARS-CoV-2 infection in mice. K18-ACE2 transgenic male mice that were $8-10$ weeks old were administered, intraperitoneally, $200 \mu \mathrm{l}$ of Dulbecco's PBS (DPBS) alone or DPBS containing oxPAPC at a dose of $2 \mathrm{mg}$ per $\mathrm{kg}$ body weight. At $1 \mathrm{~h}$ post-administration, mice were anesthetized with $5 \%$ isoflurane and then inoculated intranasally with SARS-CoV-2 in $50 \mu \mathrm{l}$ of DPBS containing around $2 \times 10^{4}$ plaque-forming units (p.f.u.). At $3 \mathrm{~d}$ post-infection, the mice were administered another dose of DPBS or DPBS + oxPAPC. Mice were weighed daily and monitored over a period of $14 \mathrm{~d}$ for survival. For BALF collection, mice were sacrificed at day 2 post-infection.

Intratracheal instillation and histopathology. Age- and sex-matched, 6- to 9-week-old male and female mice were used, and each mouse was administered $25 \mu \mathrm{g}$ of the SARS-CoV-2 E (ABclonal Technology, catalog no. RP01263) or S (ABclonal Technology, catalog no. RP01283LQ) protein (LPS contamination is $<0.1$ endotoxin unit (EU) $\mu \mathrm{g}^{-1}$ of protein, tested by the Limulus amebocyte lysate method) via intratracheal instillation. PBS and Pam3CSK4 were used as negative and positive controls, respectively. At $24 \mathrm{~h}$ post-administration, serum was collected, and lungs were fixed in $10 \%$ formalin and then embedded in paraffin using standard procedures. For immunohistochemistry, formalin-fixed, paraffin-embedded lungs were cut into $4-\mu \mathrm{m}$ sections. CD45 (BD Pharmingen, catalog no. 553076, 1:500) and terminal deoxynucleotidyl transferase dUTP nick-end labeling (TUNEL; Promega, catalog no. PRG7130) staining was performed according to the manufacturer's instructions and examined by a pathologist blinded to the experimental groups. BALF collection was performed at $6 \mathrm{~h}$ post-instillation.

Gene expression analysis from patients with COVID-19. Nanostring nCounter data were kindly shared by Hadjadj et al. ${ }^{16}$ for healthy patients and patients with moderate, severe and critical COVID-19. Expression of MYD88, TRIF, TLR1, TLR2, TLR3, TLR4, TLR5, TLR7, TLR8 and TLR9 was generated based on the average expression of selected genes using Morpheus.

Immunoblot analysis. For caspase- 1 analysis, BMDMs were lysed together with the supernatant using $50 \mu \mathrm{l}$ of caspase lysis buffer $(1 \times$ protease inhibitors, $1 \times$ phosphatase inhibitors, $10 \% \mathrm{NP}-40$, and $25 \mathrm{mM}$ dithiothreitol). Then $125 \mu \mathrm{l}$ of $4 \times$ sodium dodecylsulfate loading buffer was added for boiling the samples. For analysis of all other proteins, the supernatants of stimulated cells were discarded, and then cells were washed once with PBS, after which radioimmunoprecipitation lysis buffer was added to lyse the cells.

Electrophoresis was conducted to separate proteins in $10-12 \%$ polyacrylamide gels. Then proteins were transferred on to poly(vinylidene fluoride) membranes and blocked with $5 \%$ skimmed milk for $1 \mathrm{~h}$ at room temperature. All the primary antibodies were incubated overnight at $4{ }^{\circ} \mathrm{C}$, whereas secondary antibodies with horseradish peroxidase were incubated for $1 \mathrm{~h}$ at room temperature. Images were developed via a GE Amersham Imager 600, and final images were analyzed via Fiji for MacOS X (v.2.0.0-rc-67/1.52c).

The antibodies used were: anti-caspase-1 (AdipoGen, catalog no. AG-20B-0042, 1:2,000), anti-GSDMD (abcam, catalog no. ab209845, 1:1,000), anti-pERK (Cell Signaling Technology (CST), catalog no. 9101, 1:1,000), anti-pIкB (CST, catalog no. 2859, 1:1,000), anti-ERK (CST, catalog no. 9102, 1:1,000), anti-IKB (CST, catalog no. 9242, 1:1,000), anti- $\beta$-actin (Proteintech, catalog no. 66009-1-IG, 1:5,000), anti-glyceraldehyde 3-phosphate dehydrogenase (GAPDH) (CST, catalog no. 5174, 1:1,000) and horseradish peroxidase-conjugated secondary antibodies (Jackson ImmunoResearch Laboratories, anti-rabbit (catalog no. 111035-047), 1:5,000; anti-mouse (catalog no. 315-035-047), 1:5,000).

RT-PCR analysis. RNA extraction was conducted using TRIzol (Thermo Fisher Scientific, catalog no. 15596026). Complementary DNA synthesis was done by using the First Strand cDNA Synthesis Kit (Applied Biosystems, catalog no. 
4368814) according to the manufacturer's instructions. Real-time (RT)-PCR was performed with $2 \times$ SYBR Green (Applied Biosystems, catalog no. 4368706) on an ABI 7500 fast RT-PCR machine. Primers used were as follows: mouse-Gapdh: 5'-CGT CCC GTA GAC AAA ATG GT-3', 5' -TTG ATG GCA ACA ATC TCC AC-3'; Illb: 5'-GAT CCA CAC TCT CCA GCT GCA-3', 5' -CAA CCA ACA AGT GAT ATT CTC CAT G-3'; Il6: 5'-GAC AAA GCC AGA GTC CTT CAG AGA G-3', 5'-CTA GGT TTG CCG AGT AGA TCT C-3'; Tnf: 5' -CAT CTT CTC AAA ATT CGA GTG ACA A-3' , $^{\prime}$-TGG GAG TAG ACA AGG TAC AAC CC-3'; Nlrp3: 5' -TCA GAT TGC TGT GTG TGG GAC TGA-3', $5^{\prime}$-AGC TCA GAA CCA ATG CGA GAT CCT-3'; Ifna: 5'-GCT AGG CHY TRT GCT TTC CT-3', 5'-CAC AGR GGC TGT GTT TCT TC-3'; and If $n b$ : $5^{\prime}$-GCC TTT GCC ATC CAA GAG ATG C-3' 5' $^{\prime}$-ACA CTG TCT GCT GGT GGA GTT C-3'.

Human-GAPDH: 5' -CGG AGT CAA CGG ATT TGG TCG TAT-3', 5' -AGC CTT CTC CAT GGT GGT GAA GAC-3'; IL6: 5'-GCC TTC GGT CCA GTT GCC TT-3' $5^{\prime}$-GCA GAA TGA GAT GAG TTG TC-3'; TNF: $5^{\prime}$-ATG ACT TCC AAG CTG GCC GT-3', 5' -TCC TTG GCA AAA CTG CAC CT-3'; IL1B: 5' -CCA CAG

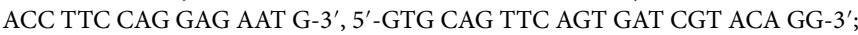
and IFNG: 5' - GAG TGT GGA GAC CAT CAA GGA AG-3' ${ }^{\prime} 5^{\prime}-$ TGC TTT GCG TTG GAC ATT CAA GTC-3'.

Cytokine analysis. For in vivo analyses and studies using BMDMs, cytokines were measured by multiplex ELISA (Millipore, catalog no. MCYTOMAG-70K) or IL-18 ELISA (Invitrogen, catalog no. BMS618-3) according to the manufacturer's instructions. For studies using PBMCs, the release of proinflammatory cytokines in the supernatant was measured by multiplex ELISA (Millipore, catalog no. HCYTMAG-60K-PX29) according to the manufacturer's instructions. For multiplex ELISAs, all the antibodies were prepared by the manufacturer.

Statistical analysis. GraphPad Prism 7.0 software was used for data analysis. Data are represented as mean \pm s.e.m. Statistical significance was determined by unpaired, two-tailed Student's $t$-test for two-group comparisons, one-way analysis of variance (ANOVA) with Dunnett's multiple comparisons test for comparisons of more than two groups, two-way ANOVA for comparisons of more than two groups with two or more timepoints, or log-rank test for survival experiments. $P$ values $<0.05$ were considered to be statistically significant, where ${ }^{\star} P<0.05,{ }^{*} P<0.01$, ${ }^{* * *} P<0.001$ and ${ }^{* * * *} P<0.0001$

Reporting Summary. Further information on research design is available in the Nature Research Reporting Summary linked to this article.

\section{Data availability}

The datasets generated and analyzed during the current study are contained within the manuscript and accompanying supplementary figures. Nanostring nCounter data were kindly shared by Hadjadj et al. ${ }^{16}$ for healthy patients and patients with moderate, severe and critical COVID-19. Source data are provided with this paper.

\section{References}

41. Kanneganti, T. D. et al. Critical role for Cryopyrin/Nalp3 in activation of caspase-1 in response to viral infection and double-stranded RNA. J. Biol. Chem. 281, 36560-36568 (2006).

42. Yamamoto, M. et al. Role of adaptor TRIF in the MyD88-independent toll-like receptor signaling pathway. Science 301, 640-643 (2003).

43. Takeuchi, O. et al. Differential roles of TLR2 and TLR4 in recognition of Gram-negative and Gram-positive bacterial cell wall components. Immunity 11, 443-451 (1999).

44. Hoshino, K. et al. Cutting edge: Toll-like receptor 4 (TLR4)-deficient mice are hyporesponsive to lipopolysaccharide: evidence for TLR4 as the LPS gene product. J. Immunol. 162, 3749-3752 (1999).
45. Hemmi, H. et al. Small anti-viral compounds activate immune cells via the TLR7 MyD88-dependent signaling pathway. Nat. Immunol. 3, 196-200 (2002).

46. Hemmi, H. et al. A toll-like receptor recognizes bacterial DNA. Nature 408, 740-745 (2000)

47. Adachi, O. et al. Targeted disruption of the MyD88 gene results in loss of IL-1- and IL-18-mediated function. Immunity 9, 143-150 (1998).

48. Gitlin, L. et al. Essential role of MDA-5 in type I IFN responses to polyriboin osinic:polyribocytidylic acid and encephalomyocarditis picornavirus. Proc. Natl Acad. Sci. USA 103, 8459-8464 (2006).

49. Suthar, M. S. et al. IPS-1 is essential for the control of West Nile virus infection and immunity. PLoS Pathog. 6, el000757 (2010).

50. Kumar, H. et al. Essential role of IPS-1 in innate immune responses against RNA viruses. J. Exp. Med. 203, 1795-1803 (2006).

51. Schickli, J. H., Zelus, B. D., Wentworth, D. E., Sawicki, S. G. \& Holmes, K. V. The murine coronavirus mouse hepatitis virus strain A59 from persistently infected murine cells exhibits an extended host range. J. Virol. 71, 9499-9507 (1997).

52. Lee, J. et al. Emergence and magnitude of ML336 resistance in Venezuelan equine encephalitis virus depend on the microenvironment. J. Virol. 94, e00317920 (2020).

\section{Acknowledgements}

We thank all the members of the Kanneganti laboratory for their comments and suggestions during the development of this article. We thank A. Burton (St Jude Children's Research Hospital) for her technical support. We also thank R. Tweedell for scientific editing and writing support and S. Surendrathanan for technical assistance. Work from our laboratory is supported by the US NIH (grant nos. AI101935, AI124346, AR056296 and CA253095 to T.-D.K.) and the American Lebanese Syrian Associated Charities (to T.-D.K.). The content is solely the responsibility of the authors and does not necessarily represent the official views of the NIH. The following reagents were deposited by the Centers for Disease Control and Prevention and obtained through BEI Resources, NIAID, NIH: SARS-Related Coronavirus 2, Isolate USA-WA1/2020, NR-52281, and Heat Inactivated, NR-52286.

\section{Author contributions}

M.Z. and T.-D.K. conceptualized the study. M.Z. and R.K. designed the methodology. M.Z., R.K., E.P.W., D.Y., E.F. and P.V. performed the experiments. M.Z. and R.K. conducted the analysis. C.B.J. provided critical reagents and scientific discussion. M.Z. and T.-D.K. wrote the manuscript with input from all authors. T.-D.K. acquired the funding and provided overall supervision.

\section{Competing interests}

The authors declare no competing interests.

\section{Additional information}

Extended data is available for this paper at https://doi.org/10.1038/s41590-021-00937-x.

Supplementary information The online version contains supplementary material available at https://doi.org/10.1038/s41590-021-00937-x.

Correspondence and requests for materials should be addressed to T.-D.K.

Peer review information Nature Immunology thanks the anonymous reviewers for their contribution to the peer review of this work. Zoltan Fehervari was the primary editor on this article and managed its editorial process and peer review in collaboration with the rest of the editorial team.

Reprints and permissions information is available at www.nature.com/reprints. 
A

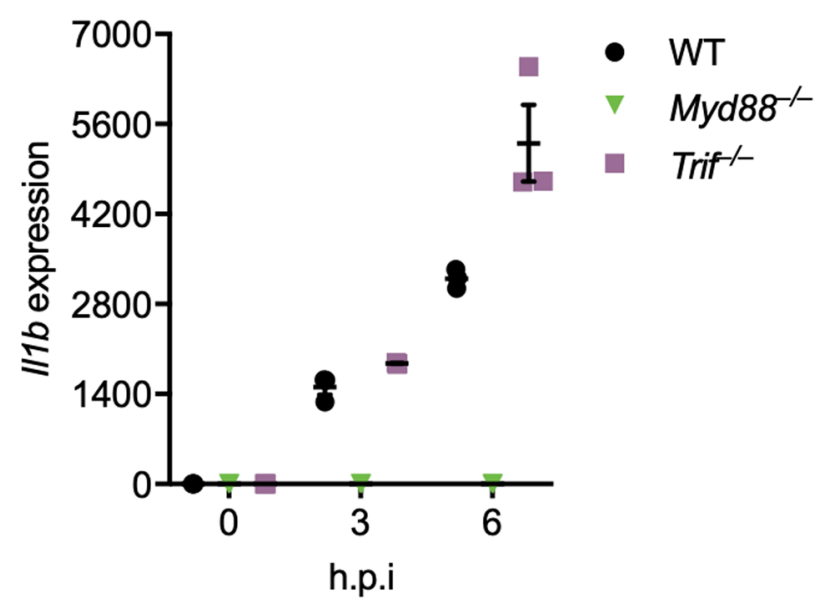

B

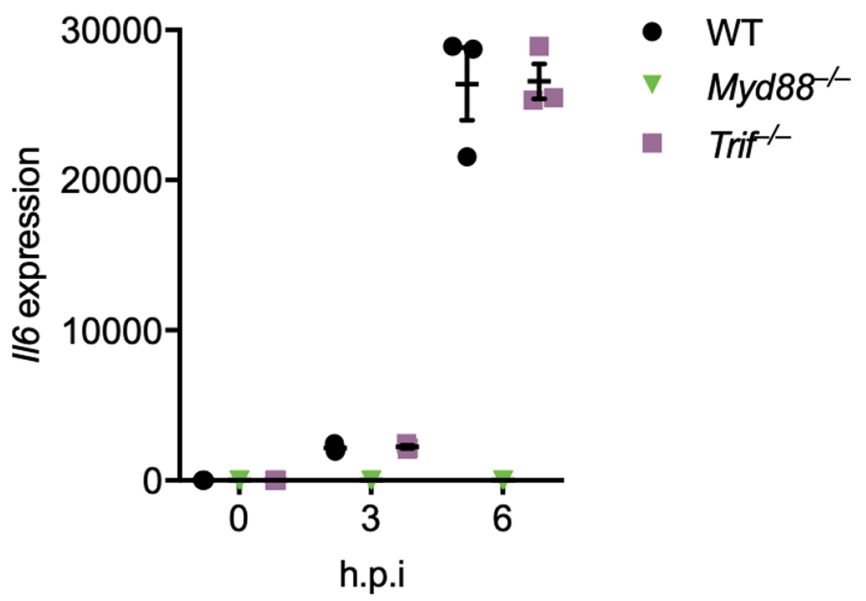

Extended Data Fig. 1 | Myd88 is required for MHV-induced inflammatory responses. (a and $\mathbf{b}$ ) Real-time PCR analysis of the expression of $/ 17 b$ (a) and 116 (b) in WT, Myd88 ${ }^{-/}$, and Trif ${ }^{-1-}$ bone marrow-derived macrophages (BMDMs) after infection with MHV at an MOI of 0.1 for the indicated time, presented relative to levels of the host gene Gapdh. Data are representative of three independent experiments. Data are shown as mean \pm SEM $(n=3)$ (a and $\mathbf{b})$. 


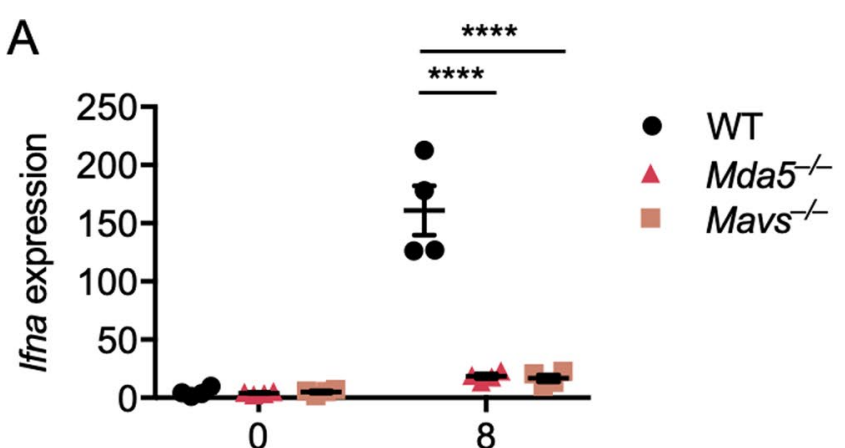

h.p.i
B

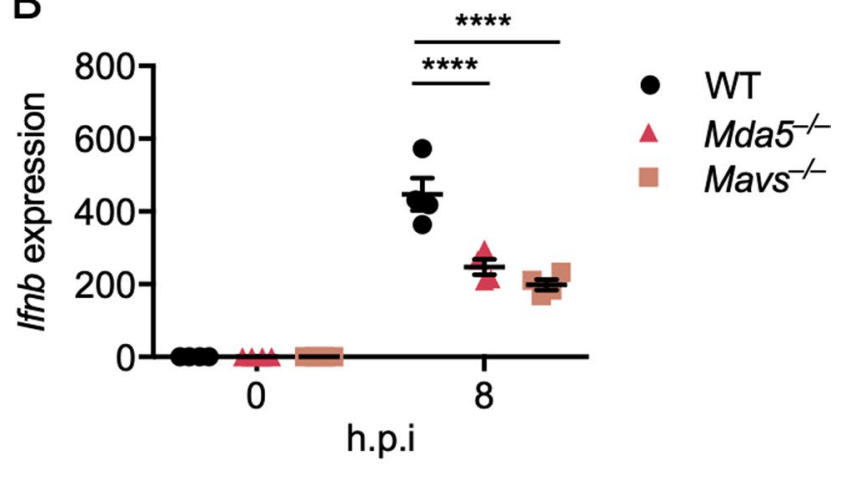

Extended Data Fig. 2 | MDA5 is required for MHV-induced type I interferon expression. (a and $\mathbf{b}$ ) Real-time PCR analysis of the expression of Ifna (a) and If $\mathrm{nb}$ (b) in WT, Mda5 $5^{-1-}$, and Mavs ${ }^{-1-}$ bone marrow-derived macrophages (BMDMs) after infection with MHV at an MOI of 0.1 for the indicated time, presented relative to levels of the host gene Gapdh. Significant differences compared to the WT group infected with MHV are denoted as ${ }^{\star * \star \star} P<0.0001$ (two-way ANOVA) (a and $\mathbf{b})$. Data are representative of three independent experiments. Data are shown as mean $\pm \operatorname{SEM}(n=3)(\mathbf{a}-\mathbf{b})$. 
A

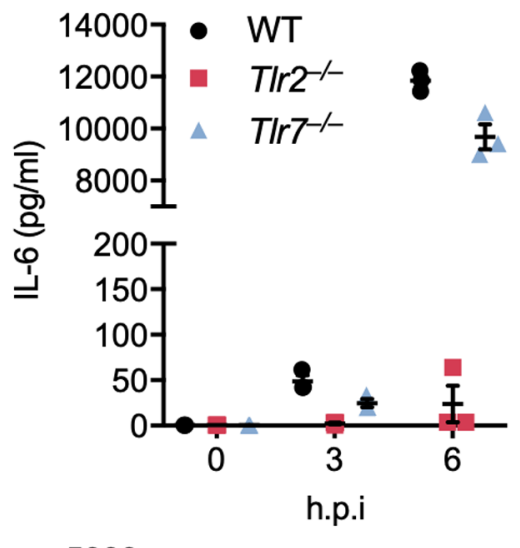

C

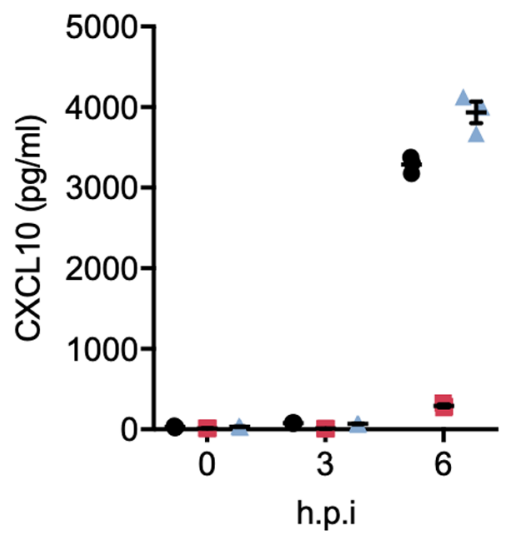

$E$

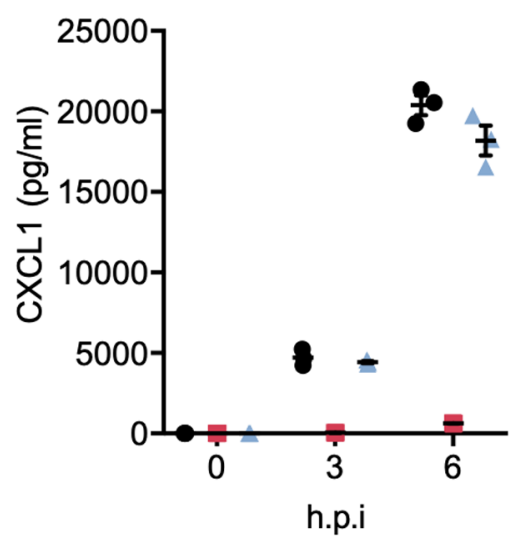

G

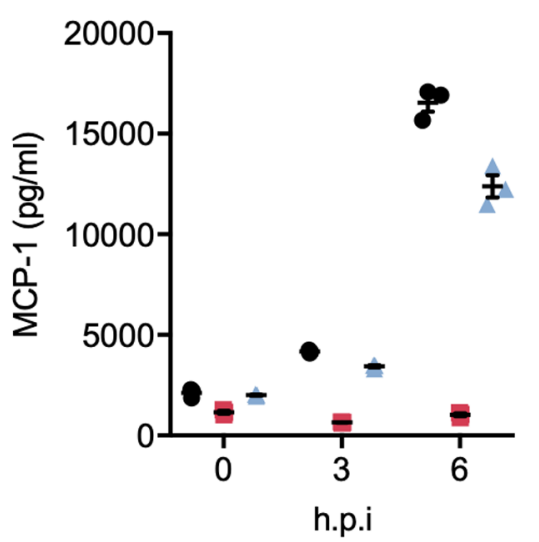

B
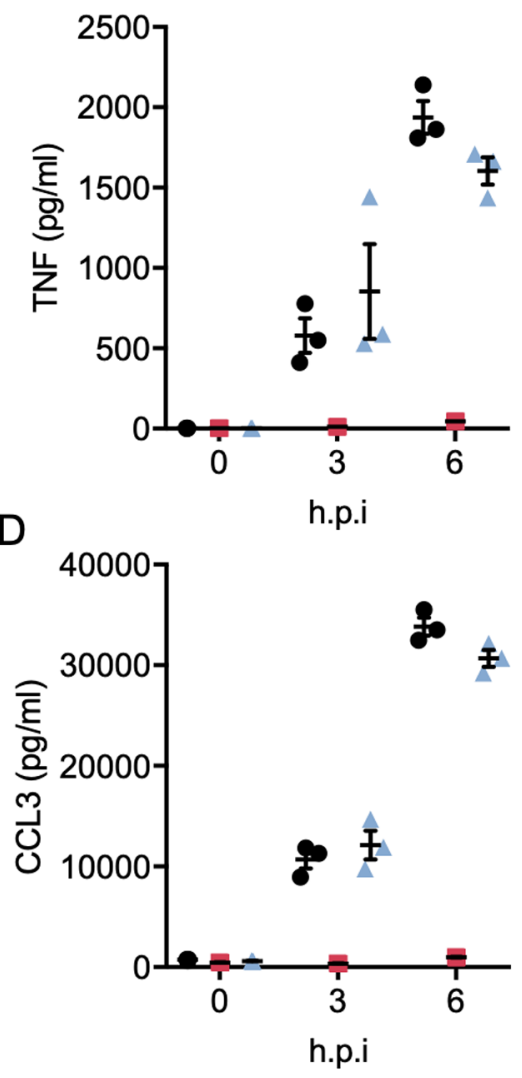

F

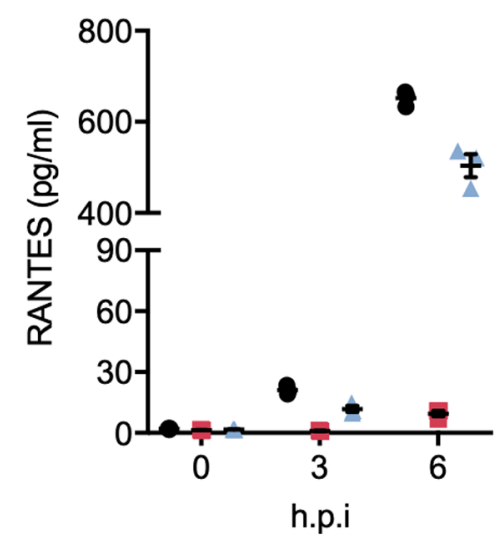

$\mathrm{H}$

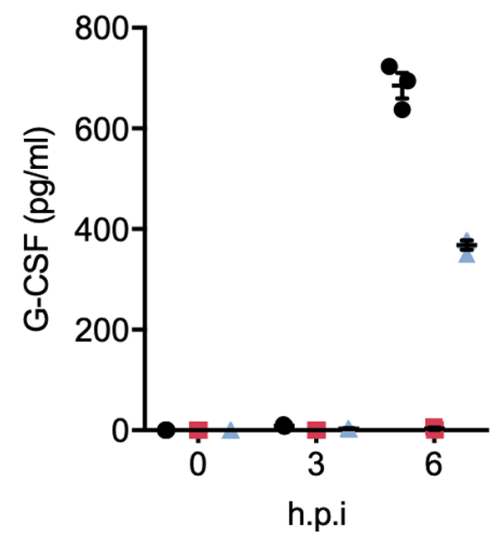

Extended Data Fig. 3 | TLR2 is essential to Inflammatory cytokine expression during MHV infection. a-h, Release of IL-6 (a), TNF- $\alpha$ (b), CXCL10 (c), $\operatorname{CCL3}(\mathbf{d}), \operatorname{CXCL1}(\mathbf{e})$, RANTES (f), MCP-1 (g), and G-CSF (h) from bone marrow-derived macrophages (BMDMs) infected with MHV at an MOI of 0.1 for the indicated time. Data are representative of three independent experiments. Data are shown as mean $\pm \operatorname{SEM}(n=3)(\mathbf{a}-\mathbf{h})$. 
A

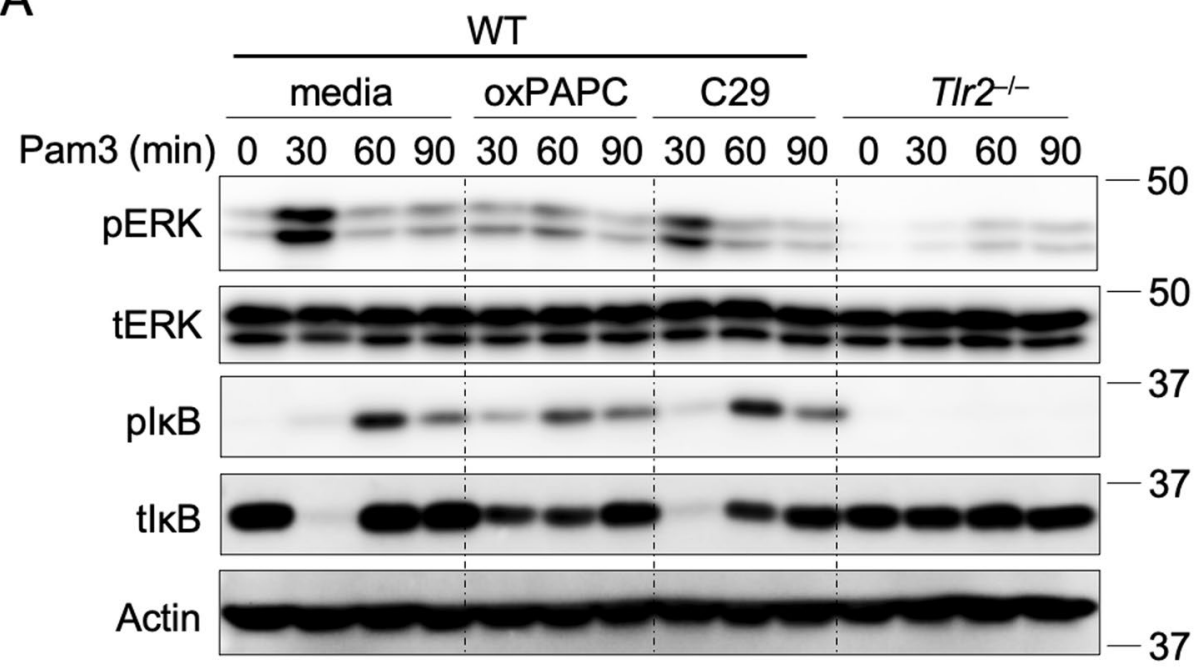

B

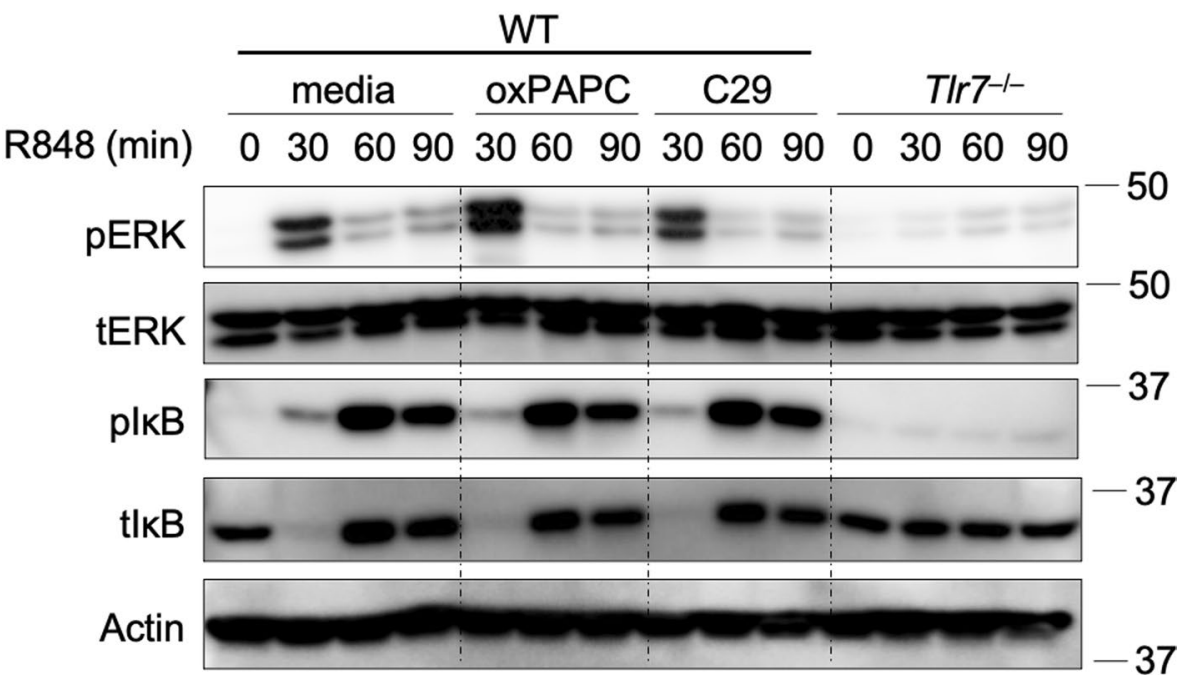

Extended Data Fig. 4 | oxPAPC is a potent TLR2 inhibitor. a, Immunoblot analysis of phospho-ERK (pERK), total ERK (tERK), plkB, and tlkB in WT and T/r2 ${ }^{-1-}$ bone marrow-derived macrophages (BMDMs) after stimulation with $1 \mu \mathrm{g} / \mathrm{ml}$ of Pam3CSK4 (Pam3) for the indicated time with or without the TLR2 inhibitor oxPAPC or C29. b, Immunoblot analysis of pERK, tERK, plkB, and t| $\mathrm{kB}$ in WT and T/r7-/- BMDMs after stimulation with $1 \mu \mathrm{g} / \mathrm{ml}$ of R848 for the indicated time with or without the TLR2 inhibitor oxPAPC or C29. Data are representative of two independent experiments. 

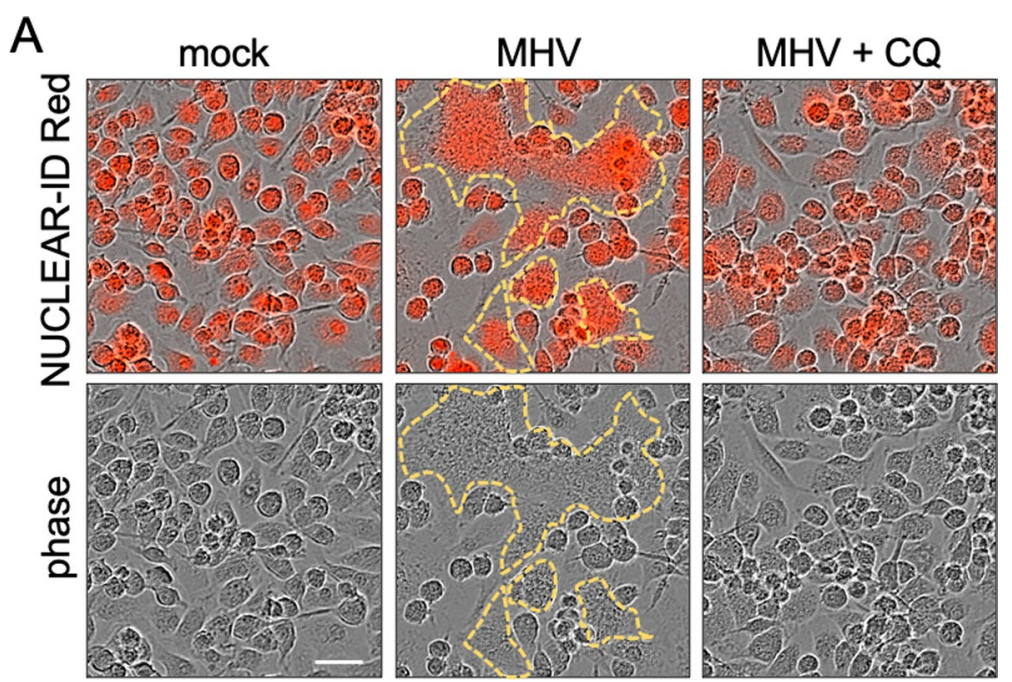

Extended Data Fig. 5 | Chloroquine can inhibit MHV-induced cell fusion. a, Cell fusion of wild type (WT) bone marrow-derived macrophages (BMDMs) after infection with MHV at an MOI of 0.1 for $8 \mathrm{~h}$ in the absence or presence of $10 \mu \mathrm{M}$ chloroquine (CQ). The upper panel was obtained via IncuCyte after staining with NUCLEAR-ID Red, and the lower panel is the corresponding phase channel. The yellow outline indicates the fused cells. Scale bar, $30 \mu \mathrm{m}$. Data are representative of three independent experiments. 
A

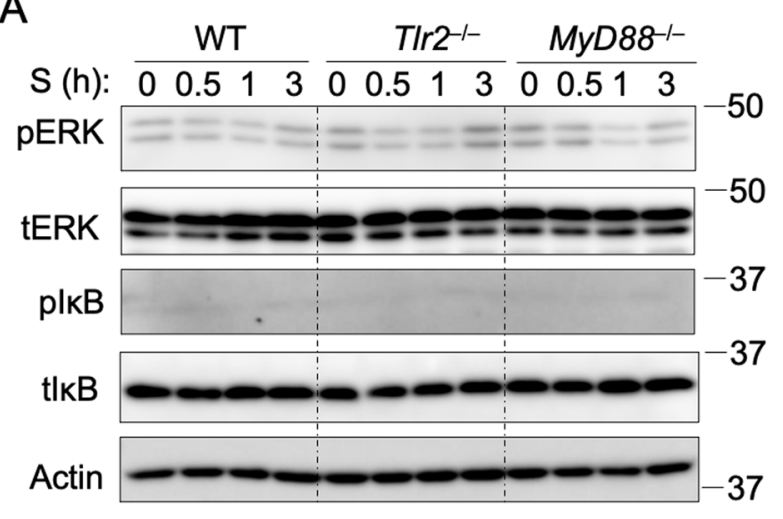

C

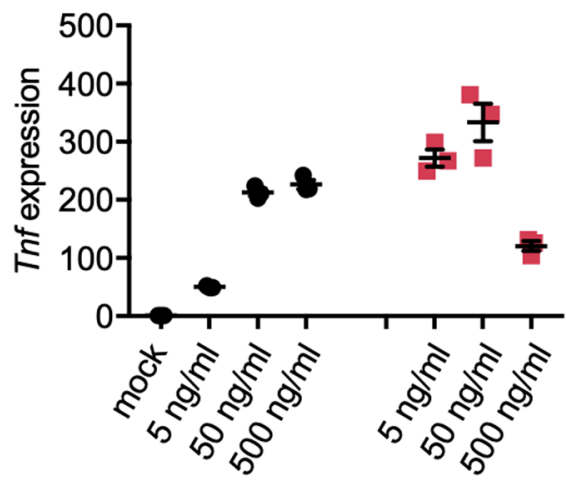

B
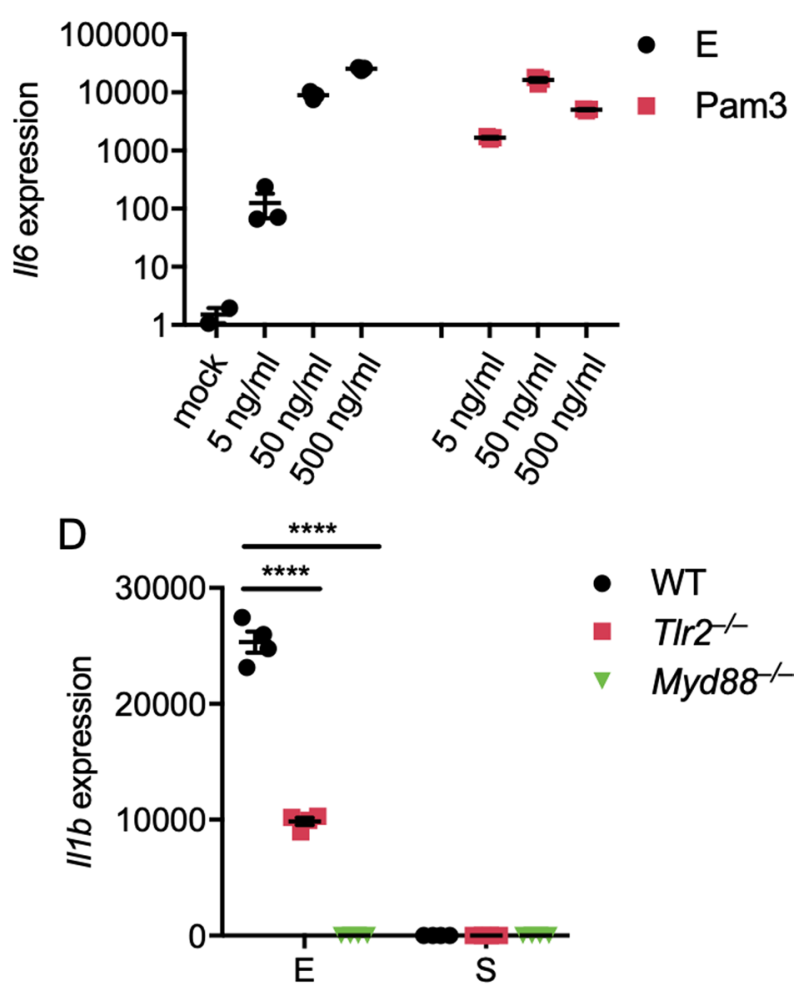

E

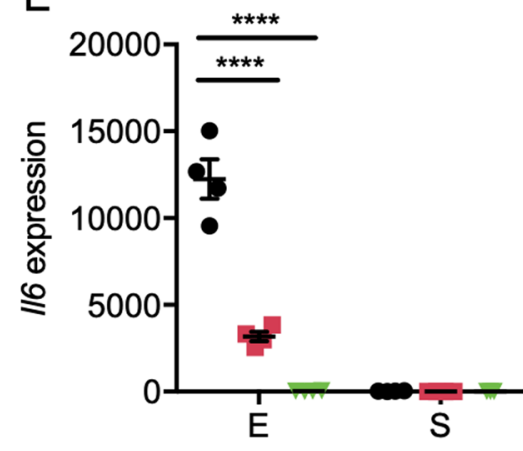

$\mathrm{H}$

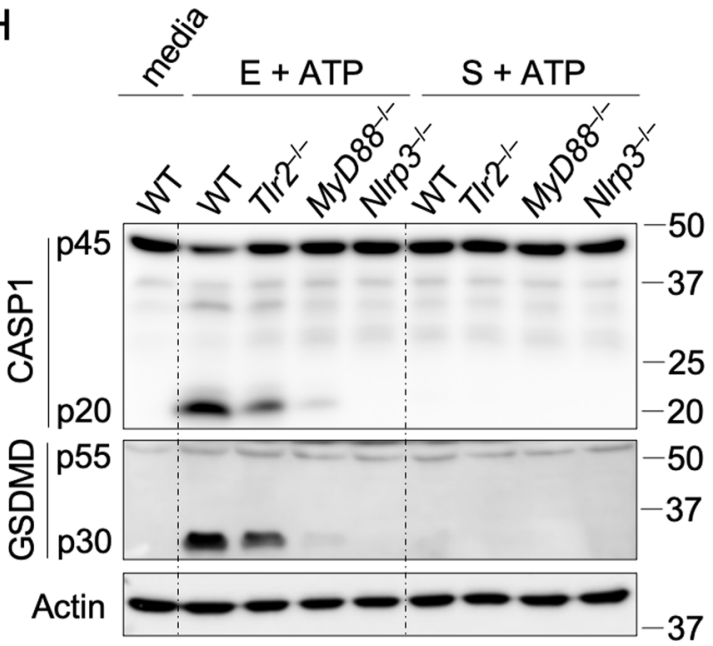

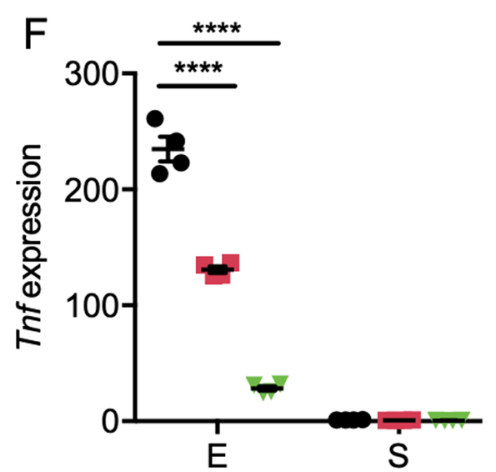

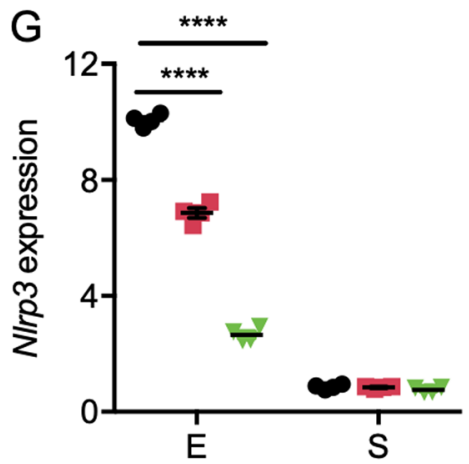

I

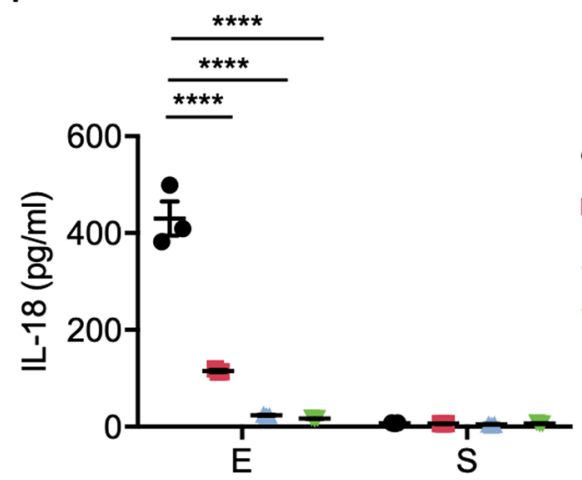

- WT

- $T / r^{2-}$

$\triangle T / r 7^{-1}$

$\checkmark \mathrm{MyD} 88^{-1-}$

Extended Data Fig. 6 | See next page for caption. 
Extended Data Fig. 6 | TLR2 can sense the envelope protein but not spike protein of SARS-CoV-2. a, Immunoblot analysis of phospho-ERK ( $p$ ERK), total

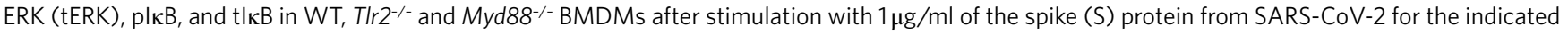
time. Actin was used as the internal control. (b and $\mathbf{c}$ ) Real-time PCR analysis of the expression of $116(\mathbf{b})$ and $\operatorname{Tnf}(\mathbf{c})$ in WT BMDMs after stimulating with the envelope (E) protein of SARS-CoV-2 or Pam3CSK4 (Pam3) for $2 \mathrm{~h}$, presented relative to levels of the host gene Gapdh. $\mathbf{d}-\mathbf{g}$, Real-time PCR analysis of

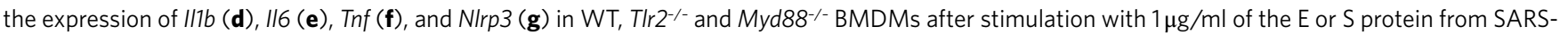
CoV-2 for $4 \mathrm{~h}$, presented relative to levels of the host gene Gapdh. $\mathbf{h}$ Immunoblot analysis of pro- (p45) and cleaved caspase-1 (p20; CASP1) and pro(p55) and cleaved gasdermin D (p30; GSDMD) in BMDMs primed with $1 \mu \mathrm{g} / \mathrm{ml}$ of the E or S protein from SARS-CoV-2 for $4 \mathrm{~h}$ and then stimulated with ATP for $45 \mathrm{~min}$. Actin was used as the internal control. i, Release of IL-18 from BMDMs after the treatment in (h). Significant differences compared to the WT group stimulated with E protein are denoted as ${ }^{\star \star \star \star} P<0.0001$ (one-way ANOVA) (d-g, i). Data are representative of two (b and $\left.\mathbf{c}\right)$ or three (a, $\left.\mathbf{d}-\mathbf{i}\right)$ independent experiments. Data are shown as mean $\pm \operatorname{SEM}(n=3)(\mathbf{b}-\mathbf{g}$ and $\mathbf{i})$. 
A S

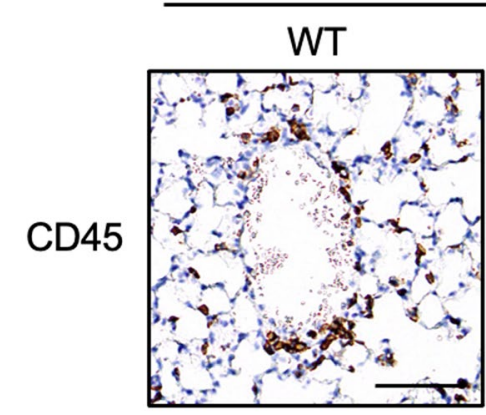

TIr $1+$
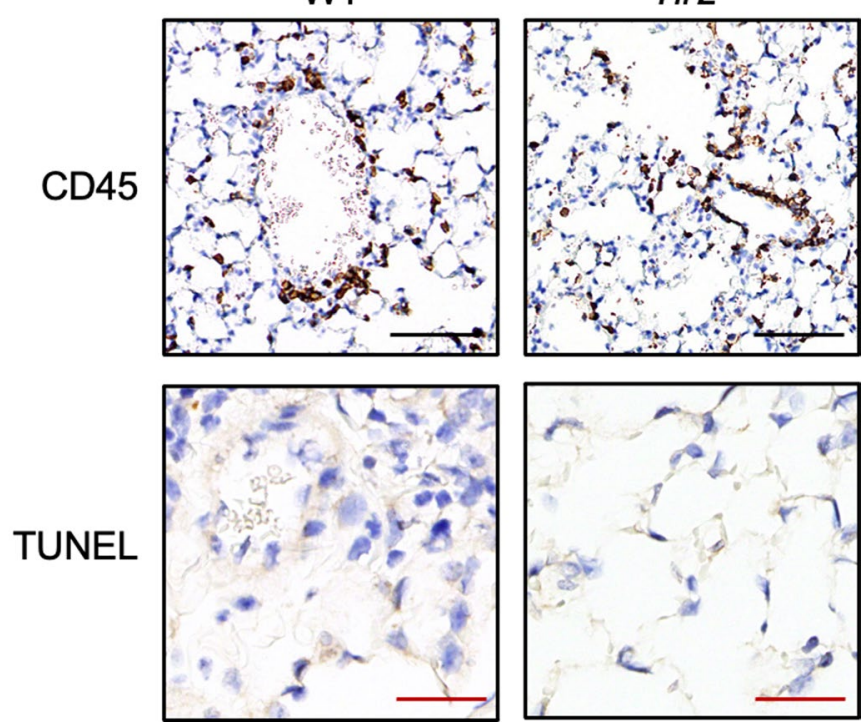

Extended Data Fig. 7 | The spike protein of SARS-CoV-2 cannot induce inflammation and damage in mouse lungs. a, CD45 immuno-staining and TUNEL staining of lung samples obtained from mice $24 \mathrm{~h}$ after being intratracheally instilled with the spike (S) protein from SARS-CoV-2. Data are representative of two independent experiments. Scale bar, $100 \mu \mathrm{m}$ (black) or $25 \mu \mathrm{m}$ (red). 


\section{Reporting Summary}

Nature Research wishes to improve the reproducibility of the work that we publish. This form provides structure for consistency and transparency in reporting. For further information on Nature Research policies, see our Editorial Policies and the Editorial Policy Checklist.

\section{Statistics}

For all statistical analyses, confirm that the following items are present in the figure legend, table legend, main text, or Methods section.

$\mathrm{n} / \mathrm{a}$ Confirmed

$\bigotimes$ The exact sample size $(n)$ for each experimental group/condition, given as a discrete number and unit of measurement

\ A statement on whether measurements were taken from distinct samples or whether the same sample was measured repeatedly

The statistical test(s) used AND whether they are one- or two-sided

Only common tests should be described solely by name; describe more complex techniques in the Methods section.

Х A description of all covariates tested

Х $\square$ A description of any assumptions or corrections, such as tests of normality and adjustment for multiple comparisons

A full description of the statistical parameters including central tendency (e.g. means) or other basic estimates (e.g. regression coefficient)

AND variation (e.g. standard deviation) or associated estimates of uncertainty (e.g. confidence intervals)

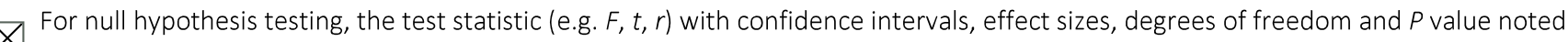
Give $P$ values as exact values whenever suitable.

Х $\square$ For Bayesian analysis, information on the choice of priors and Markov chain Monte Carlo settings

$\bigotimes \square$ For hierarchical and complex designs, identification of the appropriate level for tests and full reporting of outcomes

$\triangle \square$ Estimates of effect sizes (e.g. Cohen's d, Pearson's $r$ ), indicating how they were calculated

Our web collection on statistics for biologists contains articles on many of the points above.

\section{Software and code}

Policy information about availability of computer code

Data collection Microscopy images were collected using manufacturer supplied software (IncuCyte S3). Real-time qPCR was performed on an ABI 7500 realtime PCR instrument. For immunoblotting, membranes were developed with an Amersham imager. ELISA was conducted with MILLIPLEX Analyzer.

Data analysis For immunoblotting, images were analyzed with Fiji for MacOS X (version 2.0.0-rc-67/1.52c). Graphpad Prism version 7 were used for data analysis.

For manuscripts utilizing custom algorithms or software that are central to the research but not yet described in published literature, software must be made available to editors and

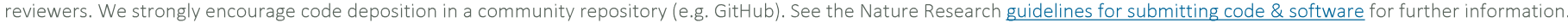

Policy information about availability of data

All manuscripts must include a data availability statement. This statement should provide the following information, where applicable:

- Accession codes, unique identifiers, or web links for publicly available datasets

- A list of figures that have associated raw data

- A description of any restrictions on data availability

The datasets generated and analyzed during the current study are contained within the manuscript and accompanying supplemental figures. Nanostring nCounter data was kindly shared by Hadjadj et al. for healthy patients and patients with moderate, severe, and critical COVID-19. 
Please select the one below that is the best fit for your research. If you are not sure, read the appropriate sections before making your selection.

\ Life sciences

Behavioural \& social sciences

Ecological, evolutionary \& environmental sciences

For a reference copy of the document with all sections, see nature.com/documents/nr-reporting-summary-flat.pdf

\section{Life sciences study design}

All studies must disclose on these points even when the disclosure is negative.

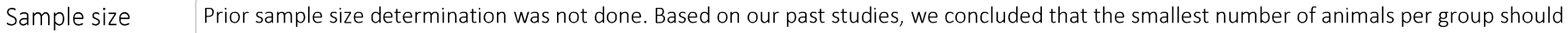
be seven for survival and body weight analysis. To arrive at this number, we used the JMP statistical package to perform a power analysis. Using parameters of alpha $=0.05$; power $=80 \%$; effect size $=50 \%$; and standard deviation $=30 \%$ of the mean, we arrived at a minimum group size of seven for a comparison between two groups.

Data exclusions $\quad$ ROUT test with Q-value of 0.1 as a cut-off was used to exclude outliers. The data exclusion criterion was pre-established. All data were retained if removing outliers could have resulted in a sample size of less than 3 . No outliers were removed from the datasets reported. For in vivo survival studies, mice that were not successfully infected were excluded. These mice were identified by monitoring body weight and clinical symptom development. Mice that lost less than $5 \%$ body weight and did not develop clinical symptoms were considered to be not infected and were excluded from the analysis.

Replication Each experiment was performed with at least 3 biological replicates. All the reported results are from experiments in which every repeat gave similar results.

Randomization Animals from the same cage were randomly selected for different treatments. For in vitro experiments, cells from the same pool of BMDMs or PBMCs were randomly split into separate wells and subjected to the treatments.

Blinding The pathologist was blinded for examination of histological analyses. For all other analyses, blinding was not performed as the experimental endpoints and readouts were quantitative and not subjective.

\section{Reporting for specific materials, systems and methods}

We require information from authors about some types of materials, experimental systems and methods used in many studies. Here, indicate whether each material, system or method listed is relevant to your study. If you are not sure if a list item applies to your research, read the appropriate section before selecting a response.

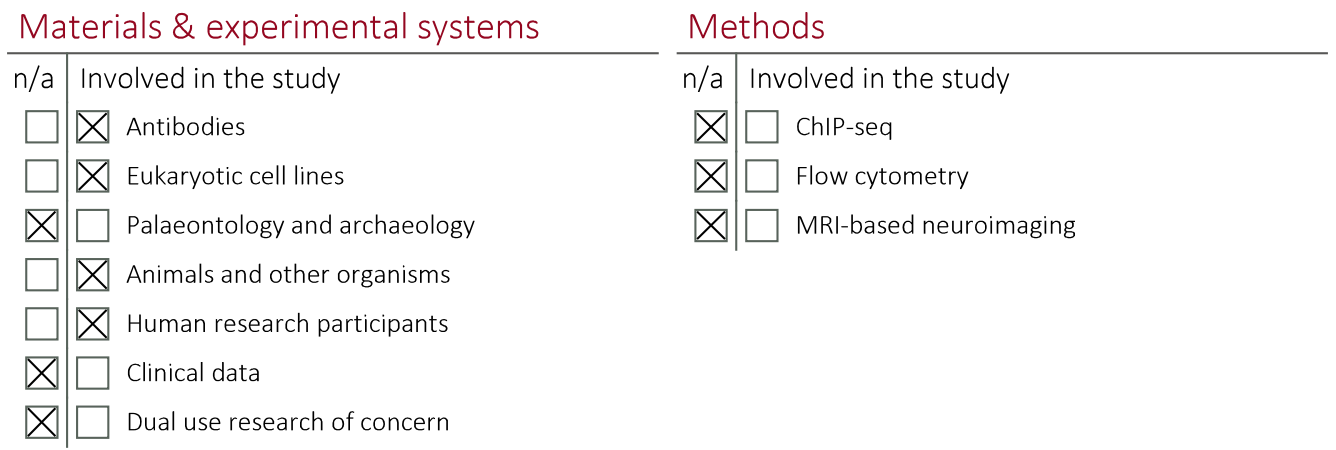

\section{Antibodies}

Antibodies used

All the antibodies used are for immunoblotting: anti-caspase-1 (AdipoGen, AG-20B-0042, 1:2000), anti-pERK (Cell Signaling Technology [CST], \#9101, 1:1000), anti-plkB (CST, \#2859, 1:1000), anti-ERK (CST, \#9102, 1:1000), anti-IKB (CST, \#9242, 1:1000), anti$\beta$-actin (Proteintech, 66009-1-IG, 1:5000), anti-GAPDH (CST, \#5174, 1:1000), anti-CD45 (BD PharmingenTM, 553076, 1:500).

Validation

All antibodies were validated by their source company. anti-caspase-1 (AG-20B-0042, Adipogen): Measuring the inflammasome: O. Gross; Methods Mol. Biol. 844, 199 (2012). https:// adipogen.com/ag-20b-0042-anti-caspase-1-p20-mouse-mab-casper-1.html anti-GSDMD (abcam, ab209845, 1:1000): Benmerzoug $S$ et al. STING-dependent sensing of self-DNA drives silica-induced lung inflammation. Nat Commun 9:5226 (2018). https://www.abcam.com/gsdmd-antibody-epr19828-ab209845.html anti-pERK (9101, Cell Signaling): Michelle D Tallquist, et. al. Additive effects of PDGF receptor beta signaling pathways in vascular smooth muscle cell development. PLoS Biol. 2003. https://www.cellsignal.com/products/primary-antibodies/phospho-p44-42-mapkerk1-2-thr202-tyr204-antibody/9101

anti-plKB (2859, Cell Signaling): Lixian Wu, et. al. IKKß regulates the repair of DNA double-strand breaks induced by ionizing radiation in MCF-7 breast cancer cells. Plos One. 2011. https://www.cellsignal.com/products/primary-antibodies/phospho-ikba-ser32-14d4rabbit-mab/2859 
anti-ERK (9102, Cell Signaling): Katrina Podsypanina, et. al. Evolution of somatic mutations in mammary tumors in transgenic mice is influenced by the inherited genotype. BMC Med. 2004. https://www.cellsignal.com/products/primary-antibodies/p44-42-mapkerk1-2-antibody/9102

anti-IKB (9242, Cell Signaling): James Schuman, et. al. A critical role of TAK1 in B-cell receptor-mediated nuclear factor kappaB activation. Blood. 2009. https://www.cellsignal.com/products/primary-antibodies/ikba-antibody/9242

anti-B-actin (66009-1-IG, Proteintech): Zeyang Ji et al. Kibra Modulates Learning and Memory via Binding to Dendrin. Cell Report. 2019. https://www.ptglab.com/products/Pan-Actin-Antibody-66009-1-lg.htm

anti-GAPDH (5174, Cell Signaling): Ashutosh Kumar et al. SWELL1 regulates skeletal muscle cell size, intracellular signaling, adiposity and glucose metabolism. Elife. 2020. https://www.cellsignal.com/products/primary-antibodies/gapdh-d16h11-xp-rabbit-mab/5174 anti-CD45 (553076, BD PharmingenTM): Johnson P, Maiti A, Ng DHW. CD45: A family of leukocyte-specific cell surface glycoproteins In: Herzenberg LA, Weir DM, Herzenberg LA, Blackwell C, ed. Weir's Handbook of Experimental Immunology, Vol 2. Cambridge: Blackwell Science; 1997; :62.1-62.16. https://www.bdbiosciences.com/eu/applications/research/stem-cell-research/cancer-research/ human/purified-rat-anti-mouse-cd45-30-f11/p/553076

\section{Eukaryotic cell lines}

Policy information about cell lines

Cell line source(s)

Vero-E6: ATCC (VERO C1008); 17Cl-1 cells were a kind gift from Dr. Rudragouda Channappanavar (UTHSC); 293T cells: ATCC (CRL-3216).

Authentication

The Vero-E6 line was verified by ATCC and monitored for cross-contamination. The $17 \mathrm{Cl}-1$ cells were monitored for morphology and verified by microscopy. The 293T line was verified by ATCC and monitored for cross-contamination.

Mycoplasma contamination

Cells were tested for mycoplasma contamination using mycoplasma detection PCR and were found to be negative for mycoplasma contamination.

Commonly misidentified lines

(See ICLAC register)

This study did not use commonly misidentified cell lines.

\section{Animals and other organisms}

Policy information about studies involving animals; ARRIVE guidelines recommended for reporting animal research

Laboratory animals

For non-infectious experiment, C57BL6/J mice or mutant mice backcrossed to this background were used. In all experiments both male and female mice between 6-9 weeks old were used. Mice were housed in 20-23.3 degrees Celsius and 30-70\% humidity with 12 $\mathrm{h}$ light/dark cycles.

For infectious experiment, 8-10-week old hACE2-transgenic C57BL6/J male mice purchased from Jackson Lab were used.

Wild animals

The study did not involve wild animals

Field-collected samples The study did not involve field collected samples.

Ethics oversight

Noninfectious animal studies were conducted under protocols approved by the St. Jude Children's Research Hospital committee on the Use and Care of Animals. SARS-CoV-2 infections were performed at the University of Tennessee Health Science Center under ABSL3 conditions in accordance with the approval of the Institutional Animal Care and Use Committee of University of Tennessee Health Science Center (Protocol \#20-0132).

Note that full information on the approval of the study protocol must also be provided in the manuscript.

\section{Human research participants}

Policy information about studies involving human research participants

Population characteristics

Whole blood was obtained from anonymous healthy donors at St. Jude Children's Research Hospital. The investigators remain blinded to the donor characteristics.

Recruitment

Anonymous voluntary healthy donors at St. Jude Children's Research Hospital were recruited. The investigators remain blinded to the details.

Ethics oversight

The blood donation center at St. Jude Children's Research Hospital operates following protocols approved by the IRB of SJCRH.

Note that full information on the approval of the study protocol must also be provided in the manuscript. 